This article has been accepted for publication in Monthly Notices of the Royal Astronomical Society (C): 2019 The Authors. Published by Oxford University Press on behalf of the Royal Astronomical Society. All rights reserved. 


\title{
Fast radio burst dispersion measures and rotation measures and the origin of intergalactic magnetic fields
}

\author{
S. Hackstein, ${ }^{1 \star}$ M. Brüggen, ${ }^{1}$ F. Vazza ${ }^{\circledR},{ }^{1,2,3}$ B. M. Gaensler ${ }^{\oplus 4,5}$ and V. Heesen ${ }^{\oplus 1}$ \\ ${ }^{1}$ Hamburger Sternwarte, University of Hamburg, Gojenbergsweg 112, D-21029 Hamburg, Germany \\ ${ }^{2}$ Department of Physics and Astronomy, University of Bologna, Via Gobetti 93/2, I-40129 Bologna, Italy \\ ${ }^{3}$ Istituto di Radioastronomia, INAF, Via Gobetti 101, I-40129 Bologna, Italy \\ ${ }^{4}$ Dunlap Institute for Astronomy and Astrophysics, University of Toronto, Toronto, ON M5S 3H4, Canada \\ ${ }^{5}$ Department of Astronomy and Astrophysics, University of Toronto, Toronto, ON M5S 3H4, Canada
}

Accepted 2019 July 12. Received 2019 July 10; in original form 2019 April 18

\begin{abstract}
We investigate the possibility of measuring intergalactic magnetic fields using the dispersion measures and rotation measures of fast radio bursts. With Bayesian methods, we produce probability density functions for values of these measures. We distinguish between contributions from the intergalactic medium, the host galaxy, and the local environment of the progenitor. To this end, we use constrained, magnetohydrodynamic simulations of the local Universe to compute lines-of-sight integrals from the position of the Milky Way. In particular, we differentiate between predominantly astrophysical and primordial origins of magnetic fields in the intergalactic medium. We test different possible types of host galaxies and probe different distribution functions of fast radio burst progenitor locations inside the host galaxy. Under the assumption that fast radio bursts are produced by magnetars, we use analytic predictions to account for the contribution of the local environment. We find that less than 100 fast radio bursts from magnetars in stellar-wind environments hosted by starburst dwarf galaxies at redshift $z \gtrsim 0.5$ suffice to discriminate between predominantly primordial and astrophysical origins of intergalactic magnetic fields. However, this requires the contribution of the Milky Way to be removed with a precision of $\approx 1 \mathrm{rad} \mathrm{m}^{-2}$. We show the potential existence of a subset of fast radio bursts whose rotation measures carry information on the strength of the intergalactic magnetic field and its origins.
\end{abstract}

Key words: polarization-galaxies: intergalactic medium-galaxies: magnetic fieldscosmology: large-scale structure of universe-cosmology: observations-radio continuum: general.

\section{INTRODUCTION}

Fast radio bursts (FRBs) are impulsive bursts in the radio sky of very short duration $(0.1-10 \mathrm{~ms})$ with frequencies of about $1 \mathrm{GHz}$, observed down to $400 \mathrm{MHz}$ (Lorimer et al. 2007). Their observed dispersion measure (DM) exceeds the contribution of the Milky Way (MW), implying an extragalactic origin. Their short duration suggests an emitting region of the order of $100 \mathrm{~km}$, suggesting a neutron star origin. Such a small region only allows for small intrinsic variation of, e.g. the polarization angle, used to observe the Faraday rotation measure (RM), which is directly related to the magnetic field strength along the line of sight (LoS). FRBs are hence potential probes for the intergalactic medium (IGM) and interstellar medium (ISM) in the MW and in the host galaxy, especially in the local environment of the FRB progenitor (see e.g. Zheng et al. 2014;

^E-mail: shackste@physnet.uni-hamburg.de
Keane et al. 2016; Ravi et al. 2016). In this work, we investigate whether FRBs with observed RMs can be used to derive information on the origin of intergalactic magnetic fields (IGMFs).

Currently, the most widely accepted constraints on the comoving strength of magnetic fields in voids stem from observations of the CMB ( $B \lesssim 4.4 \times 10^{-9} \mathrm{G}$, Ade et al. 2016) and of TeV-Blazars ( $B \gtrsim 3 \times 10^{-16} \mathrm{G}$, Neronov \& Vovk 2010), about seven orders of magnitude apart. For a summary of constraints on the magnetic field strength and coherence lengths, see Taylor, Vovk \& Neronov (2011) or Dzhatdoev et al. (2018).

A number of processes have been proposed to generate cosmic magnetic fields. Primordial scenarios consider processes in the early Universe, mostly prior to the recombination epoch, e.g. during phase transitions or inflation (e.g. Campanelli 2009; Kahniashvili et al. 2010; Subramanian 2016). Another possible scenario is the generation of magnetic fields during early galaxy formation, e.g. by feedback of active galactic nuclei (AGNs; e.g. Vazza 
et al. 2017) or winds from compact galaxies (Kronberg, Lesch \& Hopp 1999; Donnert et al. 2009; Dubois \& Teyssier 2010). For a detailed overview on the different models, see e.g. Widrow (2002). These two scenarios result in severely different predictions for the magnetic field strengths in voids of the large-scale structure. In reality, it is likely that both the scenarios contribute to the origin of cosmic magnetic fields. Measuring their strength would allow us to put reasonable constraints on the origin of those fields (e.g. Vazza et al. 2017).

Since their first discovery (Lorimer et al. 2007), there has been a large number of studies addressing the nature and origin of FRBs (e.g. Zhang 2014; Ravi \& Loeb 2018; Marcote \& Paragi 2019), see Katz (2016a), Lorimer (2018), and Petroff, Hessels \& Lorimer (2019) for reviews. Ravi et al. (2019) have summarized the expected progress in the coming decade.

So far, two repeating sources have been identified (Scholz et al. 2016; Spitler et al. 2016; CHIME/FRB Collaboration 2019) that rule out cataclysmic scenarios, at least for those events. Many more discoveries are expected in the very near future. Repeating signals allow us to test time dependence of their properties, making them the subject of intensive studies (e.g. Lu, Kumar \& Narayan 2018; Hessels et al. 2019; Houben et al. 2019; Li et al. 2019; Lyutikov 2019; Yang, Zhang \& Zhang 2019).

Still, very little is known about the population and origin of FRBs (e.g. Caleb, Spitler \& Stappers 2018; Katz 2018; Palaniswamy, Li \& Zhang 2018; Caleb et al. 2019; James 2019). To keep track of all the different models, they are collected in the living theory catalogue of FRBs (Platts et al. 2018). Here, we investigate the application of FRBs as probes of cosmic magnetism, with a few priors on their possible origin. We present a framework that can be used to compare observations to theory to make quantitative inferences.

At this point, only a few FRBs have observed RMs. Once the next generation of telescopes, such as e.g. CHIME/FRB, FAST, MeerKat, and SKA, begin their surveys, this number is expected to increase drastically (Jonas 2009; Nan et al. 2011; Keane, Fender \& Hassall 2013; Macquart et al. 2015; The CHIME/FRB Collaboration 2018).

Akahori, Ryu \& Gaensler (2016) produced predictions for the intergalactic $\mathrm{DM}_{\mathrm{IGM}}$ and $\mathrm{RM}_{\mathrm{IGM}}$ of FRBs from the IGM. They use numerical simulations for the large-scale structure and the IGMF to test whether combining both measurements yields information on IGMFs. Their results show that the RM is dominated by the hot gas in clusters while the dominant contributor to DM changes with redshift. Still, they show that the radial component of the densityweighted IGMF strength in filaments can be inferred from combined measurements within a factor of $\sim 2$.

Vazza et al. (2018) investigate the variance in $\mathrm{RM}_{\mathrm{IGM}}$ due to the assumed magneto-genesis model. They assume astrophysical or primordial origin of cosmic magnetic fields, similar to the models used in this work. For FRBs located at a redshift of $z=1$, they find differences in $\left\langle\mathrm{RM}_{\mathrm{IGM}}\right\rangle$ between the models of about one order of magnitude. In principle, this allows us to draw conclusions on the strength of the IGMF. However, it is unclear at which redshift the observed signal reveals most information.

Walker, Ma \& Breton (2018) provide a framework similar to the one presented in this paper. They obtain predictions of DM in the form of likelihood functions for the different contributing regions. They use these to derive estimates on the redshift of FRBs, which mostly agree with $z \approx 0$ in the lower bounds. Thus, they conclude that the observed $\mathrm{DM}_{\text {obs }}$ can only be used to infer an upper limit on the redshift. This is in agreement with several other studies on that topic (Dolag et al. 2015; Luo et al. 2018; Niino 2018; Pol et al.

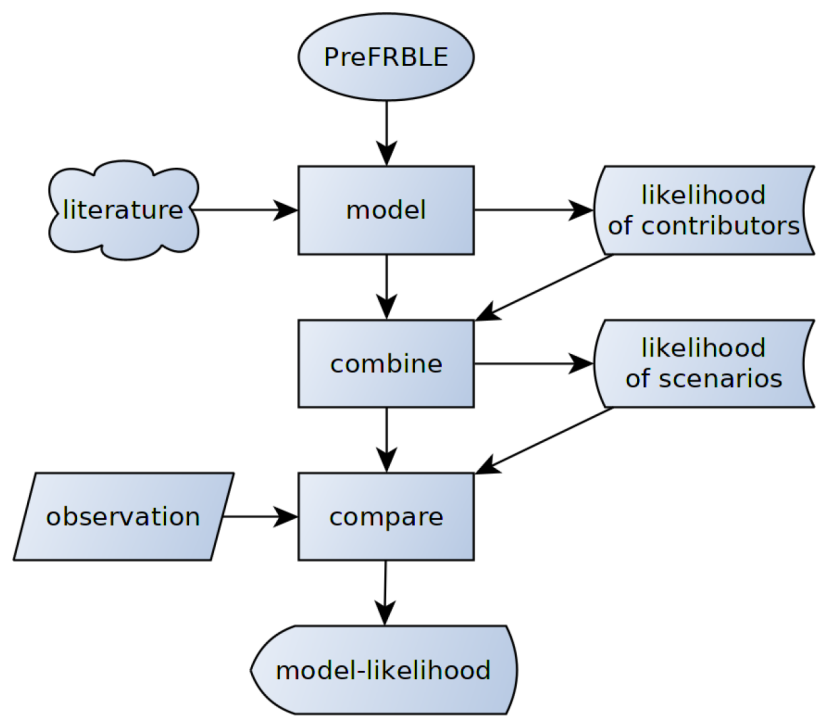

Figure 1. Flowchart to depict the basic structure of the inference presented in this work. We use results in the literature to model the contributions to DM and RM from different regions along the LoS of FRBs. These results are combined to predict the full measures expected at the Earth in different scenarios for the combination of contributor models. Finally, the results are compared to observational data to quantify and compare the posterior likelihood of several scenarios to produce the observed data.

2019). We note that the framework presented here easily allows one to infer the redshift of an FRB from its DM, similar to the findings of Walker et al. (2018) and Pol et al. (2019). However, our results are subject to the same uncertainties and cannot improve on previous results.

In this work, we show how to combine predictions of DM and $\mathrm{RM}$ for different regions along the LoS of FRBs and to compare them to the observed $\mathrm{DM}_{\mathrm{obs}}$ and $\mathrm{RM}_{\mathrm{obs}}$ in order to study the IGMF. Fig. 1 shows an overview of the basic structure of the inference.

We improve on previous studies by use of constrained simulations of the local Universe that resemble different scenarios of magnetogenesis, and further, by comparing the individual contributions to $\mathrm{DM}$ and $\mathrm{RM}$ along the $\mathrm{LoS}$, considering redshifts out to $z=6$.

This paper is organized as follows. In Section 2, we explain how we model the different contributions to DM and RM along the LoS of FRBs and how to compute their likelihood functions. In Section 3, we discuss the results of the individual models of the contributing regions. In Section 4, we combine the predictions of all contributors to predict observed $\mathrm{DM}_{\mathrm{obs}}$ and $\mathrm{RM}_{\mathrm{obs}}$. We show how these can be used to interpret $\mathrm{DM}_{\mathrm{obs}}$ and $\mathrm{RM}_{\mathrm{obs}}$ regarding the origin of IGMFs. Finally, in Section 5 we discuss our results and in Section 6 we conclude.

\section{MODELS}

In this section, we describe the models investigated in this work and how we obtain the likelihood functions. A summary of all models can be found in Table 1 .

\subsection{Intergalactic medium}

\subsubsection{Model}

We model the IGM using constrained magnetohydrodynamic (MHD) simulations of the local Universe, produced with the ENZO 
Table 1. Summary of all models investigated in this work. $n_{\mathrm{FRB}}$ is the assumed number density of possible progenitor positions, and $n_{\star}$ is the number density of stars in the MW. NE2001 stands for the density model of thermal electrons in the MW presented in Cordes \& Lazio (2002). JF12 stands for the magnetic field model of the MW developed by Jansson \& Farrar (2012).

\begin{tabular}{ll}
\hline Mnemonic & Physics \\
\hline IGM: & \\
Primordial & 3D-MHD model of the local Universe with strong uniform initial magnetic field of $B_{0}=1$ nG comoving \\
Astrophysical & 3D-MHD model of the local Universe with very weak initial magnetic field and magnetic feedback of an AGN
\end{tabular}

Host galaxy:

$\begin{array}{ll}\text { Uniform } & \text { MW-like spiral galaxy model (NE2001 and JF12), } n_{\mathrm{FRB}}=\text { const. } \\ \text { Star density } & \text { MW-like spiral galaxy model (NE2001 and JF12), } n_{\mathrm{FRB}} \propto n_{\star} \\ \text { Dwarf } & \text { Starburst dwarf galaxy similar to IC10 or host of FRB121102, } n_{\mathrm{FRB}} \propto n_{\star}\end{array}$

Local environment of progenitor:

$\begin{array}{ll}\text { Uniform } & \text { Magnetar in uniform ISM environment } \\ \text { Wind } & \text { Magnetar in environment dominated by stellar winds of seed star } \\ \text { Wind }+ \text { SNR } & \text { Wind } \text { plus contributions of SNR }\end{array}$

Milky Way:

NE2001 + JF12 Best-fitting model for Galactic RM (NE2001 and JF12)

code (Bryan et al. 2014). The simulations start from initial conditions obtained from a procedure summarized by Sorce et al. (2016). The constraints applied in order to reproduce the local Universe at $z=0$ are fully described by Tully et al. (2013). Simulations have been produced within the Planck cosmology framework $\left(\Omega_{\mathrm{m}}=\right.$ 0.307, $\Omega_{\Lambda}=0.693, h=0.677, \sigma_{\mathrm{s}}=0.829$, Planck Collaboration I 2014). Further information on the models can be found in Hackstein et al. (2018), where they have been investigated in the context of propagation of cosmic rays. The three-dimensional data sets at $z$ $=0$ are also publicly available at https://crpropa.desy.de/ under 'Additional resources'.

We consider two different scenarios for the predominant genesis of IGMFs, primordial versus astrophysical. To do so, we make use of the result of a single simulation, which considers the primordial origin of IGMFs. From that and from the astrophysical model presented in Hackstein et al. (2018), we extract the $|B| \propto \rho$ relation in Fig. 2 (cf. Vazza et al. 2017). The difference in $|B|(\rho)$ between the two models is most prominent at very low density, far away from the central cluster regions, where most AGNs reside. However, the contribution from these regions to the RM is likely far below the ionospheric foreground $\approx 1 \mathrm{rad} \mathrm{m}^{-2}$, hence not observable. The most interesting regions are the vicinity of clusters, filaments, and other regions, where $1<\rho /\langle\rho\rangle<200$.

The primordial model starts with a uniform magnetic field with comoving magnetic field strength $B_{0}=1 \mathrm{nG}$, slightly below upper limits of the PLANCK collaboration, $B_{0} \lesssim 4.4 \mathrm{nG}$ (Ade et al. 2016). Note that Trivedi, Subramanian \& Seshadri (2014) derived an upper limit of $B_{0} \lesssim 0.6 \mathrm{nG}$ using the CMB Trispectrum. The astrophysical model is initialized with a $B_{0}=10^{-8} \mathrm{nG}$. Though this is below the lower limits obtained for present fields in voids, the final result of the simulation agrees with that limit, $B_{\text {void }} \gtrsim 3 \times 10^{-7} \mathrm{nG}$ (Neronov \& Vovk 2010). In order to obtain magnetic fields that agree with observations of clusters, this model allows for additional seeding of magnetic fields by feedback of AGNs below redshift 4 . In order to obtain results for the astrophysical model from the data of primordial, we apply the ratio of average $|B|(\rho)$ for these two models as correction factor on the magnetic field outside of galaxy clusters, where cosmic gas density $\rho<200\langle\rho\rangle$. This allows us to test different prescriptions for three-dimensional magnetic fields in our cosmological volume with a limited consumption of computing

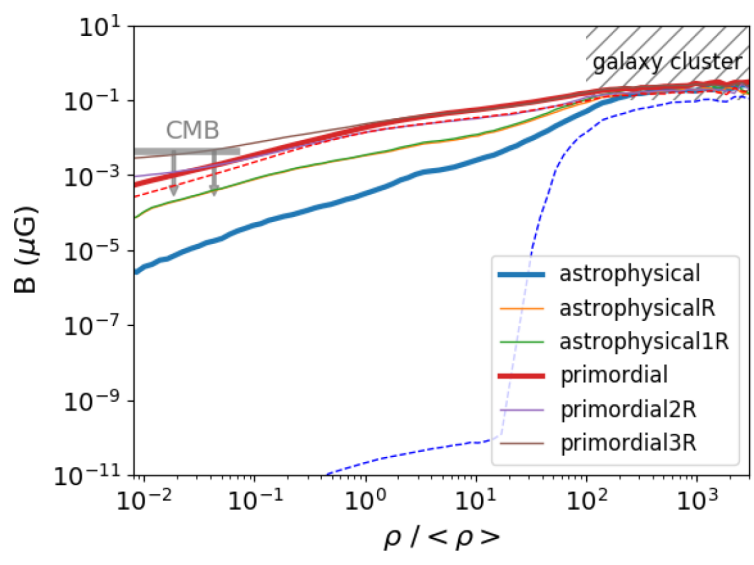

Figure 2. Relation between average magnetic field strength $B$ and gas density $\rho$ in the different IGM models investigated in Hackstein et al. (2018). The two models used in this paper are drawn with thick lines. The dashed lines show results for the median of $B$, instead of the mean. We indicate the range of magnetic fields in clusters (e.g. Feretti et al. 2012) as well as the upper limit of fields in voids according to CMB observations by PLANCK, $B_{0} \lesssim 4.4 \mathrm{nG}$ (Ade et al. 2016).

time. However, in this work we investigate only two models at the extreme ends of possible strengths of the IGMF in order to see whether FRBs carry any information on the IGMF.

Note that the average of $|B|$ is dominated by the high values in a density bin. The median, shown as dashed lines in Fig. 2, reflects much better the huge difference in the magnetic field outside highdensity structures. Using the median ignores possible high values of $|B|$ within a density bin, hence underestimates the magnetic field and the RM. The average instead is dominated by these high values and forces the magnetic field to values of similar strength, everywhere within the density bin. In this case, the results for the astrophysical model are much closer to the primordial, representing the most pessimistic case to tell the two extreme models apart. Hence, the use of the average instead of the median strengthens the conclusion that the observation of FRBs can be used to distinguish between these two models. 
We note, however, that the primordial model we probe here is initialized with a uniform field, whose topology is preserved in lowdensity regions. This can affect the distribution of RM from FRBs in the local Universe, making smaller values less probable. This is because the contributions from different parts of the IGM are less likely to cancel out each other. In Appendix A, we investigate how that influences the final results and find a negligible impact on observable $\mathrm{RM}_{\mathrm{obs}} \gtrsim 1 \mathrm{rad} \mathrm{m}^{-2}$ (see also Vazza et al. 2018).

The use of numerical simulations will improve our results over those of Walker et al. (2018) and Niino (2018) by accounting for the uncertainty that arises due to inhomogeneities in the IGM. Like Akahori et al. (2016), we apply the usual method of cosmological data stacking (e.g. da Silva et al. 2000). We reconstruct the cosmic space from redshift $z=0$ to 6 with use of simulation outputs at redshifts $z=0.0,0.2,0.5,1.5,2.0,3.0$, and 6.0. The LoS starts at redshift $z=6$ and traverses the simulated volume in a randomly oriented rectilinear path. When the LoS reaches the corresponding redshift, the trajectory is continued in the next snapshot. The final snapshot at $z=0$ is used from half the cosmic time towards the previous snapshot at $z=0.2$. Finally, all values are corrected for a smooth evolution with redshift.

The $\mathrm{DM}_{\mathrm{IGM}}$ for a LoS with cosmological distance is

$\mathrm{DM}_{\mathrm{IGM}}=\int_{0}^{d_{\mathrm{FRB}}}(1+z)^{-1}\left(\frac{n_{\mathrm{e}}}{\mathrm{cm}^{-3}}\right)\left(\frac{\mathrm{d} l}{\mathrm{pc}}\right) \mathrm{pc} \mathrm{cm}^{-3}$,

where $d_{\mathrm{FRB}}$ is the comoving distance to the FRB source and $n_{\mathrm{e}}$ is the proper thermal electron density (Xu \& Han 2015). DM measures the extra traveltime of radiation at low frequencies due to dispersive effects in plasma. Hence, it scales with redshift as $\mathrm{DM} \propto(1+z)^{-1}$.

The $\mathrm{RM}_{\mathrm{IGM}}$ for a LoS with cosmological distance is

$$
\begin{aligned}
\mathrm{RM}_{\mathrm{IGM}}= & \frac{\Delta \Phi}{\Delta \lambda^{2}} \approx 0.81 \int_{d_{\mathrm{FRB}}}^{0}(1+z)^{-2}\left(\frac{B_{\|}}{\mu \mathrm{G}}\right)\left(\frac{n_{\mathrm{e}}}{\mathrm{cm}^{-3}}\right) \\
& \times\left(\frac{\mathrm{d} l}{\mathrm{pc}}\right) \mathrm{rad} \mathrm{m}^{-2},
\end{aligned}
$$

where $B_{\|}$the proper magnetic field component parallel to the LoS (Xu \& Han 2014). RM is the ratio of relative rotation of polarization angles $\Phi$ at different frequencies divided by the difference of the squared wavelength $\lambda$. The former is not affected by cosmic expansion, therefore $\mathrm{RM}$ scales with redshift as $\mathrm{RM} \propto(1+z)^{-2}$.

The free electron density, $n_{\mathrm{e}}$, is computed assuming full ionization and a mean molecular weight $\mu_{\mathrm{e}} \approx 1.16$ of an electron for cosmic fractions of hydrogen and helium.

\subsubsection{Likelihood function}

We obtain the likelihood function of the IGM contribution from LoS integrals. These are produced using the LIGHTRAY function of the TRIDENT package (Hummels, Smith \& Silvia 2017). This function extracts field values from data cells intersected by a LoS, defined by start and end positions in the three-dimensional volume. It also computes the redshift that reflects the distance to the observer. This way, it allows us to compute the redshift-corrected values along the LoS that contribute to the DM and RM.

These LoSs start from the position of the MW, defined as the centre of the box in our constrained simulation of the local Universe. They progress in evenly distributed directions defined by the HEALPIX (Górski et al. 2005) tessellation of the sphere (similar to Stasyszyn et al. 2010). We use a total of $49152 \mathrm{LoSs}$, corresponding to a pixel radius of $1-2^{\circ}$. This allows us to resolve local structures while computation costs are kept at a minimum. The total computation took about $1200 \mathrm{~h}$ of CPU time. Differences in the likelihood function are $<0.1$ per cent compared to the next smaller tessellation of the sky with 12288 LoSs. Hence, the likelihood function is reasonably converged.

The total path-length of the LoS exceeds the size of the constrained high-resolution portion of our simulation volume, which is the central $(250 \mathrm{Mpc})^{3}$. Therefore, direction-dependent results beyond the first crossing of this region would be misleading. Instead, for results at higher redshifts $(z \gtrsim 0.1)$ we investigate a statistical sample of LoS with random orientation. These are obtained by stacking segments between random points taken from the constrained regions, until the LoS reaches the redshift of the current snapshot. The process continues with the next snapshot, until the full $\operatorname{LoS}$ is built. The final snapshot of the simulation is at $z$ $=0$ and would not be used in the procedure described above. Hence, it is used until half of the cosmic time towards the next snapshot at $z=0.2$, where $z \approx 0.9$. The fact that $\nabla \cdot \vec{B}$ is not conserved to 0 at the interfaces where we combine different segments of the LoS does not pose any problem for our analysis, as $<1$ per cent of cells are affected by this problem.

The likelihood function is proportional to the amount of LoSs that deliver the same value. Assuming an isotropic distribution of FRBs in the sky, the calculation is straightforward:

$p\left(\mathrm{DM}^{\prime} \mid z\right)=\frac{\oint \delta\left(\mathrm{DM}(\theta, \phi ; z)=\mathrm{DM}^{\prime}\right) \mathrm{d} \theta \mathrm{d} \phi}{\oint \mathrm{d} \theta \mathrm{d} \phi} \approx \frac{N_{\mathrm{DM}^{\prime}}}{N_{\mathrm{tot}}}$,

where $N_{\mathrm{DM}^{\prime}}$ is the number of LoSs from a redshift $z$ with $\mathrm{DM} \approx$ $\mathrm{DM}^{\prime}$, and $N_{\text {tot }}$ is the total number of LoSs from that redshift. The same holds for the RM.

\subsection{Host galaxy}

\subsubsection{Model}

To highlight the influence of host galaxies, we investigate two different types of galaxies, a spiral galaxy similar to the MW and a starburst dwarf galaxy similar to the host of FRB121102. We note that this small number of models does not suffice to reflect the variety of different galaxies that are likely to host FRBs, but gives a rough estimate on the range of possible contributions.

Integrating the galaxy stellar mass function (Baldry et al. 2012) yields that 68 per cent of stars reside in galaxies of $10^{11}-10^{12} \mathrm{M}_{\odot}$, similar to the MW. Such galaxies are likely hosts, if FRBs are produced by magnetars (e.g. Popov \& Postnov 2010; Katz 2016b; Beloborodov 2017; Metzger, Berger \& Margalit 2017; Metzger, Margalit \& Sironi 2019). We obtain predictions for the spiral host galaxy with use of the NE2001 model (Cordes \& Lazio 2002) for the thermal electron density, combined with the JF12 model (Jansson \& Farrar 2012) for the magnetic field, where we use the best-fitting parameters for the MW. Luo et al. (2018) compared the results of NE2001 with the model of Yao, Manchester \& Wang (2017) and found that the overall statistics are rather similar, NE2001 tending to slightly higher values of DM. Here we exclusively use the NE2001 model, which was also assumed by Jansson \& Farrar (2012).

Though it has been argued that the NE2001 model is not good enough to exactly reconstruct the DM foreground of pulsar data (see Xu \& Han 2015), it is a reasonable choice to obtain a decent statistical estimate. Calculations have been performed using the HAMMURABI code (Waelkens et al. 2009), which computes the LoS integrals in evenly distributed directions on the whole sky seen from a given position to the edge of the galaxy model. 
The likelihood of a given value of $\mathrm{DM}_{\text {host }}$ and $\mathrm{RM}_{\text {host }}$ from the host highly depends on the position of the progenitor within the host, which is uncertain. To account for that, a reasonable choice is to sample different possible positions and combine their predictions. The positions are drawn randomly, following a probability density that we assume to be either uniform or proportional to the star density. In particular, for the latter we use the combination of a thick disc and a thin disc of radius $R_{i}$ and scale height $Z_{i}$ with exponentially falling star density

$n_{\text {star }}(R, Z) \propto \exp \left(-\frac{R}{R_{i}}-\frac{|Z|}{Z_{i}}\right)$,

using the best-fitting parameters obtained for distribution of $\mathrm{M}$ dwarfs in the MW, i.e. $R_{\text {thick }}=3.6 \mathrm{kpc}, Z_{\text {thick }}=0.9 \mathrm{kpc}, R_{\text {thin }}$ $=2.6 \mathrm{kpc}$, and $Z_{\text {thin }}=0.3 \mathrm{kpc}$ (Jurić et al. 2008).

Dwarf irregular galaxies in a starburst phase, which we will refer to as starburst dwarf galaxies hereafter, have high star formation rates, hence their stellar population is relatively young. Magnetars have short lifespans, $\approx 10^{4} \mathrm{yr}$ (e.g. Beniamini et al. 2019), and are produced by massive stars, $20-45 \mathrm{M}_{\odot}$ (Chabrier 2003), that have rather short lifetimes, $\sim 10^{7} \mathrm{yr}$ (e.g. Wit et al. 2005). This makes starburst dwarf galaxies a likely host for FRBs produced by magnetars.

The first localized FRB121102 was indeed found to reside in such a starburst dwarf galaxy, having a high star formation rate, low metallicity, and no AGN (Chatterjee et al. 2017; Tendulkar et al. 2017). Low-mass and low-metallicity galaxies with high star formation rates were also found to be overrepresented hosts of gamma-ray bursts and superluminous supernovae at low redshift (e.g. Fruchter et al. 2006; Vergani et al. 2015; Perley et al. 2016).

A well-studied starburst dwarf galaxy in the local Universe is IC 10 , which is at $0.8 \mathrm{Mpc}$ distance. It is the only member of the Local Group that is currently in the starburst phase. Its properties are very similar to that of the host of FRB121102 (e.g. Richer et al. 2001; Leroy et al. 2006; Magrini \& Gonçalves 2009). We use the magnetic field model of Heesen et al. (2011), who studied IC 10 with radio continuum polarimetry, to estimate the possible RM contribution of a starburst dwarf galaxy. We assume a constant thermal electron density $n_{\mathrm{e}}$ in the galactic mid-plane, which falls off exponentially with height. For the magnetic field, a combination of a spiral plane-parallel field and a poloidal vertical field both with a characteristic strength $B_{\text {host }}$ is used. We neglect random components of the magnetic field since they do not significantly affect the distribution of RM. The distribution of stars in dwarf galaxies is centred on the disc. We model their distribution with an exponential with a scale height of $300 \mathrm{pc}$ (similar to Leroy et al. 2006, who studied IC 10).

\subsubsection{Likelihood function}

Within the MW-like spiral galaxy, we draw a sample of possible positions of the progenitor, according to the assumed distribution function. Tests showed that 1000 positions are enough to ensure converged results. For each of these positions, we compute the full sky of $\mathrm{DM}_{\text {host }}$ and $\mathrm{RM}_{\text {host }}$ measurements, similar to the approach used by Walker et al. (2018). The LoS integral is then computed to the edge of the host in all different directions defined by the HEALPIX (Górski et al. 2005) tessellation of the sphere. The probability density of values on the full sky delivers the likelihood functions $P\left(\mathrm{DM}_{\text {host }}\right)$ and $P\left(\mathrm{RM}_{\text {host }}\right)$. The sum of the likelihood functions at the different positions is then the full likelihood function for the host galaxy.
Note that the results at the position of the Sun can be used to obtain predictions for the contribution from the MW itself. By construction, the results are identical to results in Jansson \& Farrar (2012).

For the starburst dwarf galaxy, we compute LoS integrals for different inclination angles and penetration depths, such that the assumed distribution of FRBs in the host is constant throughout the disc. Since the model is rotationally invariant, variations of the azimuthal angle are redundant. LoSs are calculated until they leave the disc, excluding contributions of the galactic halo, which, however, is expected to be at least one order of magnitude below the galactic contribution.

To account for possible variance across the distribution of similar starburst dwarf galaxies, we combine predictions for several choices of $n_{\mathrm{e}}$ and $B_{\text {host }}$, according to prior distributions explained in detail in Appendix B.

The dispersion delay produced at the host increases, due to cosmic expansion. The observed contribution of $\mathrm{DM}_{\text {host }}$ to the total $\mathrm{DM}_{\mathrm{obs}}$ scales with the source redshift as $(1+z)^{-1}$ (e.g. Macquart et al. 2015), so the likelihood function shifts accordingly (Walker et al. 2018) as

$p\left(\mathrm{DM}_{\text {host }} \mid z_{\mathrm{s}}\right)=\left(1+z_{\mathrm{s}}\right) p\left(\left(1+z_{\mathrm{s}}\right) \mathrm{DM}_{\text {host }} \mid z_{0}\right)$.

For $\mathrm{RM}=\frac{\Delta \Phi}{\Delta \lambda^{2}}$, the contribution of the host scales with $(1+z)^{-2}$ instead. Therefore, the corresponding likelihood function shifts as

$p\left(\mathrm{RM}_{\text {host }} \mid z_{\mathrm{s}}\right)=\left(1+z_{\mathrm{s}}\right)^{2} p\left(\left(1+z_{\mathrm{s}}\right)^{2} \mathrm{RM}_{\text {host }} \mid z_{0}\right)$.

\subsection{Local environment}

\subsubsection{Model}

We assume FRBs to be produced by magnetars (e.g. Popov \& Postnov 2010; Pen \& Connor 2015; Katz 2016b; Beloborodov 2017; Metzger et al. 2017; Metzger et al. 2019). Neutron stars are generally considered one of the most likely sources for FRBs. Beniamini et al. (2019) concluded that 12-100 per cent of neutron stars are born as magnetars. Hence, it is expected that they are numerous around star-forming regions. Their number density scales with the star formation rate. Results of Niino (2018) and Locatelli et al. (2019) suggest that the number density of FRBs does also scale with the star formation rate. This makes magnetars a likely candidate for the source of FRBs. Many other objects have been proposed as sources of FRBs (see Platts et al. 2018, who provide a living catalogue of theories). We restrict this study to exemplary compare two models of the local environment of the FRB progenitor.

To account for the local environment of a magnetar FRB progenitor, we make use of the models and results of Piro \& Gaensler (2018). They give theoretical predictions for the $\mathrm{DM}_{\text {prog }}$ and $\mathrm{RM}_{\text {prog }}$ from the local environment of the FRB, assuming they are produced by a young neutron star. They consider two models. One model assumes a uniform local ISM, while the other accounts for changes in the ISM due to stellar winds of the seed star.

In this work, we consider the two models for the uniform and wind cases, plus we consider the additional contribution of the SNR environment for the latter model in the wind $+S N R$ case. We use this low number of models to show how multiple progenitor models can be compared and combined to be tested against observations.

Stellar winds cause the magnetic field of the local environment to align and form a significant large-scale component. The RM predictions for that environment in the wind model are thus much more robust than for the supernova remnants. The latter model 


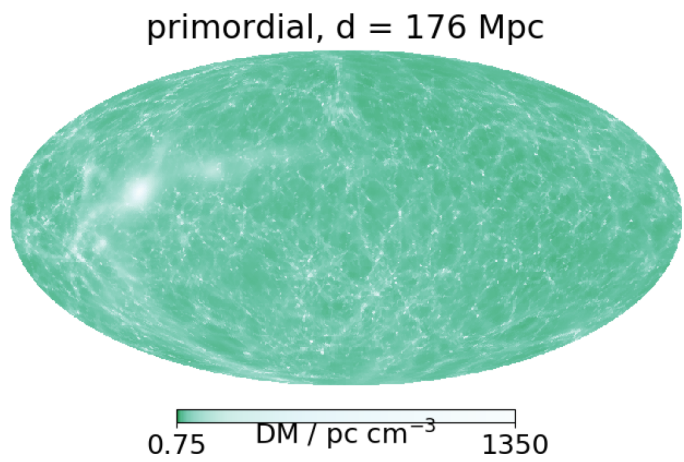

Figure 3. Full-sky map of $\mathrm{DM}_{\mathrm{IGM}}$ predictions for sources at $176 \mathrm{Mpc}$ distance in the local Universe. Results are shown in supergalactic coordinates for the primordial model. The distribution of free electrons, hence DM, is identical to the astrophysical case.

assumes the shock-generated magnetic field to be coherent, while the topology is very likely random. Hence, results for the uniform and the wind $+S N R$ model should be considered as upper limits.

\subsubsection{Likelihood function}

Under the assumption that FRBs are produced at young neutron stars, Piro \& Gaensler (2018) have derived expectation values for the $\mathrm{DM}_{\text {prog }}$ and $\mathrm{RM}_{\text {prog }}$ of the local FRB environment. These are given as functions of the ISM number density $n_{\text {ISM }}$, the time since the SN $t$, the energy of the explosion $E$, the mass of SN ejecta $M$, the wind mass-loading parameter $K$, the stellar radius $R_{\star}$, and the stellar magnetic field $B_{\star}$.

The likelihood function is obtained with a Monte Carlo method, where we sample these parameters with reasonable prior distributions, calculate the corresponding $\mathrm{DM}_{\text {prog }}$ and $\mathrm{RM}_{\text {prog }}$, and compute their probability density. The priors chosen to obtain those are summarized in Appendix B.

The contribution from the progenitor undergoes the same evolution with redshift as the contribution from the host, see equations (5) and (6).

\section{MODEL RESULTS}

\subsection{IGM, constrained results for the local Universe}

\subsubsection{Dispersion measure}

In Fig. 3, we show the full-sky projection of the expected $\mathrm{DM}_{\mathrm{IGM}}$ of FRBs at a distance of $176 \mathrm{Mpc}$. This nicely shows the distribution of structure in the local Universe (see Hackstein et al. 2018). The Virgo cluster is the most dominant local contributor with up to $\mathrm{DM}_{\mathrm{IGM}} \gtrsim$ $10^{3} \mathrm{pc} \mathrm{cm}^{-3}$.

The $\mathrm{DM}_{\mathrm{IGM}}$ prediction is the same in both IGM models, as they result in almost identical distribution of gas.

Taken from such full-sky maps at different redshifts, in Fig. 4 we present the evolution of the likelihood function of $\mathrm{DM}_{\mathrm{IGM}}$. These results agree reasonably well with results in Dolag et al. (2015) and Walker et al. (2018). At short distances, the tail at high values is more pronounced, caused by nearby, high-density regions. With increasing distance, the distribution moves towards a lognormal distribution, the mean of which increases steadily due to the cumulative growth of $\mathrm{DM}_{\mathrm{IGM}}$. Also, an increasing number of $\mathrm{LoS}$ crosses high-density structures, which add to the tail at high values.

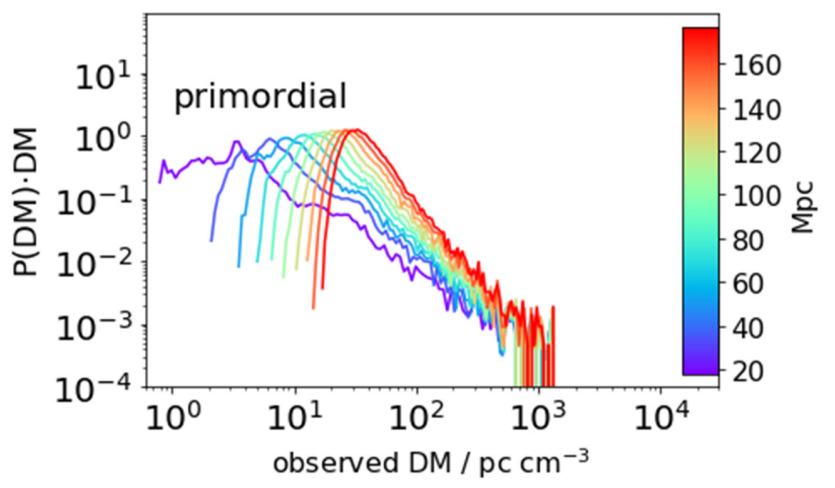

Figure 4. Likelihood function $P\left(\mathrm{DM}_{\mathrm{IGM}} \mid d\right)$ for FRB sources at distance $d$ in the local Universe, $d \lesssim 176 \mathrm{Mpc}$, for the primordial model. The distribution of free electrons, hence DM, is identical to the astrophysical case.
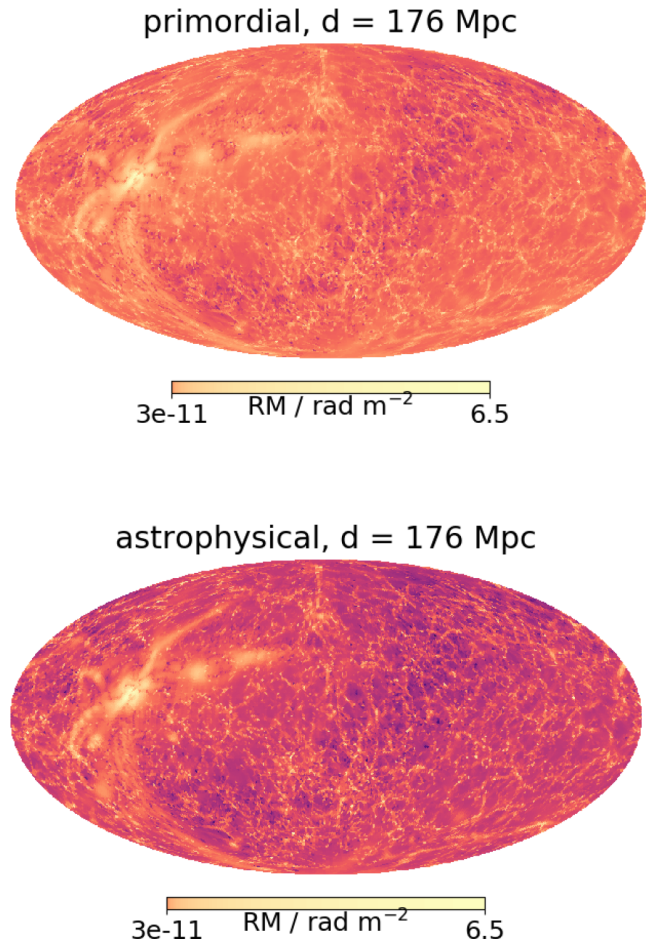

Figure 5. Full-sky map of $\left|\mathrm{RM}_{\mathrm{IGM}}\right|$ predictions for sources at $176 \mathrm{Mpc}$ distance in the local Universe, for the primordial (top) and astrophysical models (bottom). Results are shown in supergalactic coordinates.

\subsubsection{Rotation measure}

In Fig. 5, we show the full-sky projection of expected $\mathrm{RM}_{\mathrm{IGM}}$ of FRBs at a distance of $176 \mathrm{Mpc}$ for both the primordial and astrophysical models. The structure of the local Universe is nicely reproduced. Again, the Virgo cluster appears as the most dominant contributor with up to $\mathrm{RM}_{\mathrm{IGM}} \gtrsim 6 \mathrm{rad} \mathrm{m}^{-2}$, which roughly agrees with the observations of Vallée (1990).

Both IGM models result in almost identical maximum values of $\mathrm{RM}_{\mathrm{IGM}}$. These are found in LoSs that pass through regions of high density, like the Virgo cluster, that contribute very high values of $\mathrm{RM}_{\mathrm{IGM}}$. The models were built to reproduce the conditions observed in these regions in the local Universe. However, the two 


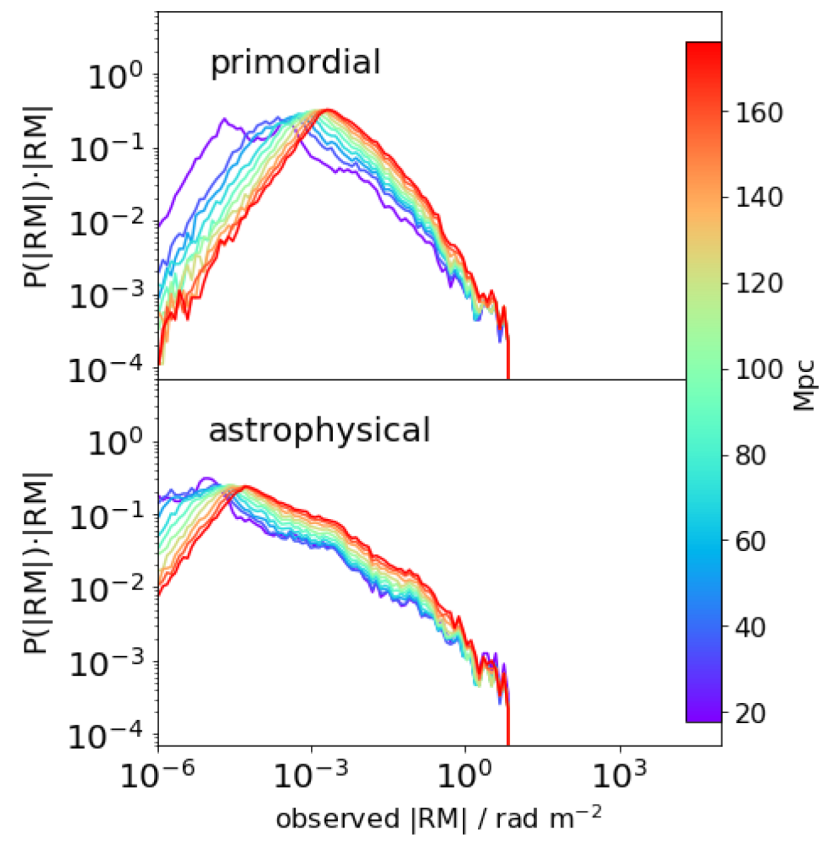

Figure 6. Likelihood function $P\left(\mid \mathrm{RM}_{\mathrm{IGM}} \| d\right)$ for FRB sources at distance $d$ in the local Universe, $d \lesssim 176 \mathrm{Mpc}$ for the primordial (top) and astrophysical models (bottom).

magneto-genesis scenarios result in severely different magnetic fields in voids. Fig. 5 shows that LoSs that do not pass through regions of high density have $\mathrm{RM}_{\mathrm{IGM}}$ lower by up to two orders of magnitude.

Taken from such full-sky maps at different redshifts, in Fig. 6 we present the evolution of the likelihood function of $\mathrm{RM}_{\mathrm{IGM}}$. Since the distribution of positive and negative values is very similar, we make use of $\log (|\mathrm{RM}|)$ in all our likelihood functions to compare contributions of different orders in more detail.

The highest values, $\mathrm{RM}_{\mathrm{IGM}} \approx 1-10 \mathrm{rad} \mathrm{m}^{-2}$, agree in both models. These are LoSs that intersect high-density regions, associated with the $\rho /\langle\rho\rangle \geq 10^{2}$ overdensity of galaxy clusters, contributing high values of RM $\mathrm{IGM}_{\mathrm{IGM}}$. However, the fraction of such LoSs is limited, and they do not affect much the overall distribution of RM IGM $_{\text {(Vazza }}$ et al. 2018).

As the peak increases with distance, the astrophysical case peaks about two orders of magnitude below the primordial case. However, the overall contribution of $\mathrm{RM}_{\mathrm{IGM}}$ is much too low to have significant influence on the total $\mathrm{RM}_{\mathrm{obs}}$ within maximum distance in the constrained volume, $176 \mathrm{Mpc}$. This also holds for possibly different results for positive and negative $\mathrm{RM}_{\mathrm{IGM}}$ caused by dense structure outside of cores of clusters.

Note that the primordial model started from a magnetic field that was coherent over the whole simulation volume. Outside of dense structures, this topology of the initial field is conserved and results in very optimistic estimates of $\mathrm{RM}_{\mathrm{IGM}}$, as contributions from separate parts of the LoS cannot cancel each other. A more detailed study of this effect can be found in Appendix A. Note that for the constrained distance, this feature is of the order of $10^{-2} \mathrm{rad} \mathrm{m}^{-2}$ in the primordial case, subdominant to other contributions along the LoS and hence not observable. At greater distances, we combine separate trajectories with random orientations, thus enabling the contributions from separate sections of the LOS to cancel each other.

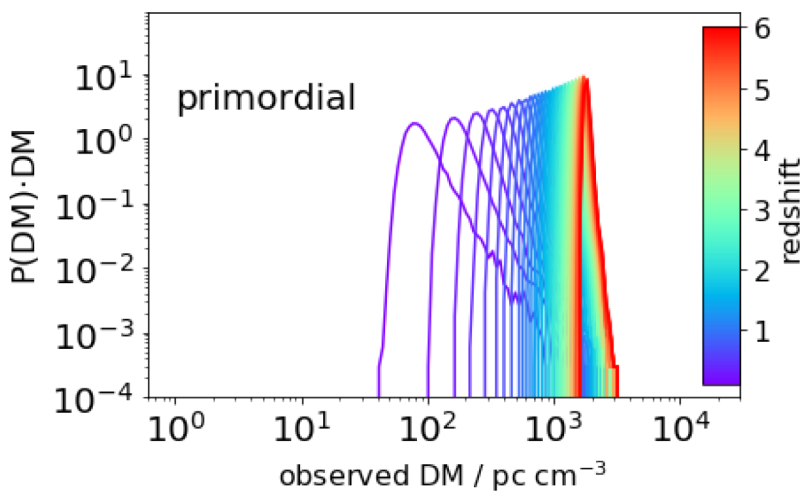

Figure 7. Likelihood function $P\left(\mathrm{DM}_{\mathrm{IGM}} \mid z\right)$ for FRB sources at redshift $z$ in the distant Universe for the primordial model. The distribution of free electrons, hence DM, is identical to the astrophysical case. From blue to red, the graphs show results at redshifts $z=0.1-6.0$ in steps of 0.1 .

\subsection{IGM, high-redshift results}

\subsubsection{Dispersion measure}

In Fig. 7, we present the resulting likelihood function of $\mathrm{DM}_{\mathrm{IGM}}$ contribution from the IGM for FRB at different redshifts in the distant Universe for the primordial model. The distribution of free electrons, hence DM, is identical to the astrophysical case. The distribution of $\mathrm{DM}_{\mathrm{IGM}}(z=1)$ is very peaked around $1000 \mathrm{pc} \mathrm{cm}^{-3}$, in good agreement with results of previous studies, where this value is reported to be $855-1200 \mathrm{pc} \mathrm{cm}^{-3}$ (Ioka 2003; Inoue 2004; McQuinn 2013; Deng \& Zhang 2014; Dolag et al. 2015; Walker et al. 2018; Pol et al. 2019). The shape is similar to the results in Fig. 4 at the highest distance and compares well with the results of Dolag et al. (2015) and Walker et al. (2018).

With increasing redshift, the proper density of free electrons increases, as does the average $\mathrm{DM}_{\mathrm{IGM}}$ contribution of the IGM. This makes the whole likelihood function $P\left(\mathrm{DM}_{\mathrm{IGM}}\right)$ shift to higher values with increasing redshift. As the cumulative growth of $\mathrm{DM}_{\mathrm{IGM}}$ from low-density regions approaches the scale of dense structure contributions, $P\left(\mathrm{DM}_{\mathrm{IGM}}\right)$ becomes much narrower. However, the overall change is slower at higher redshift $z$ (Zheng et al. 2014). Therefore, the likelihood function for high $\mathrm{DM}_{\mathrm{IGM}}$ is much broader in $z$. This shows that, although the DM delivers good upper limits on $z$, the uncertainties in the estimate will always remain rather large, and other ways to infer $z$, e.g. by identification of the host, are preferred (cf. Dolag et al. 2015; Walker et al. 2018; Kumar \& Linder 2019; Pol et al. 2019).

\subsubsection{Rotation measure}

In Fig. 8, we present the likelihood function of $\mathrm{RM}_{\mathrm{IGM}}$ contribution from the IGM for FRB at redshift $z$ in the primordial and astrophysical models of the distant Universe. As the models used here were produced with the same tools and physics as the ones used by Vazza et al. (2018), the results we find are quite similar. However, the average value of these distributions is significantly lower than the results of Akahori et al. (2016), which is due to the lower magnetic field strength outside of clusters. Here we use $B \sim 0.1 \mathrm{nG}$ instead of the 10-100 nG of Akahori et al. (2016), due to the more efficient dynamo amplification assumed in the latter model.

At lower redshifts, $z \sim 0.1, \mathrm{RM}_{\mathrm{IGM}}$ tends to low values close to zero. Only a few LoSs show high values of up to $\mathrm{RM}_{\mathrm{IGM}} \sim 100 \mathrm{rad}$ 


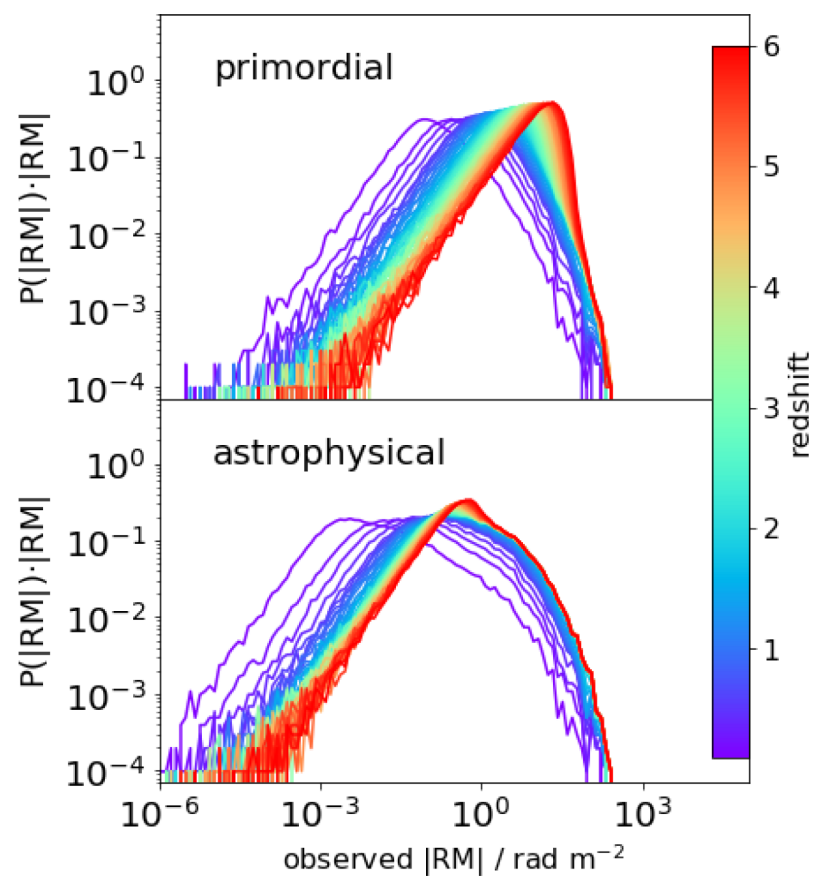

Figure 8. Likelihood function $P\left(\mathrm{RM}_{\mathrm{IGM}} \mid z\right)$ for FRB sources at redshift $z$ in the distant Universe in primordial (top) and astrophysical (down) models. From blue to red, the graphs show results at redshifts $z=0.1-6.0$ in steps of 0.1 .

$\mathrm{m}^{-2}$. These are LoSs that traverse high-density regions, associated with the $\rho /\langle\rho\rangle \geq 10^{2}$ overdensity of galaxy clusters, which contain amplified magnetic fields. With higher redshift, more and more LoS traverse clusters, some even twice, and their $\mathrm{RM}_{\mathrm{IGM}}$ reach values above $100 \mathrm{rad} \mathrm{m}^{-2}$ in both the primordial and astrophysical cases.

Many of the LoSs traverse the low-density IGM only and contribute most of $\mathrm{RM}_{\mathrm{IGM}}$. The cumulative growth shifts $\mathrm{RM}_{\mathrm{IGM}}$ to higher values, but slower than $\mathrm{DM}_{\mathrm{IGM}}$, as $\mathrm{RM}_{\mathrm{IGM}}$ from different regions of the IGM can cancel each other.

The IGM model we used considers an initial magnetic field that is coherent over $250 \mathrm{Mpc} \mathrm{h}^{-1}$, i.e. the full simulation volume. This is well conserved in low-density regions and results in a uniform sign of $\mathrm{RM}_{\mathrm{IGM}}$ contributions along a continuous LoS segment. However, since several of these segments with random orientation are combined to obtain the full LoS, they can cancel each other and we obtain results that are statistically equivalent to a stochastic field with lower coherence length.

There is a significant difference in $P\left(\mathrm{RM}_{\mathrm{IGM}} \mid z\right)$ between the primordial and astrophysical cases. The peak of $\mathrm{RM}_{\mathrm{IGM}}$ is two orders of magnitude lower in the latter case, similar to results at low redshift, shown in Fig. 6. Further, the shape looks increasingly different at higher redshift $z$. Though the peak value is rather low, $\lesssim 10 \mathrm{rad} \mathrm{m}^{-2}$ still at $z=6$, the different shapes will likely reflect in the distribution of extragalactic $\mathrm{RM}_{\mathrm{EG}}$, given that there is no dominant contribution from the other regions.

\subsection{Progenitor environment, host galaxy, and MW}

\subsubsection{Dispersion measure}

The likelihood functions of DM for all models investigated in this work are presented at redshift $z=1$ in Fig. 9.
The two models for the IGM, primordial and astrophysical, have identical $\mathrm{DM}_{\mathrm{IGM}}$ by construction. The two behaviours overlap each other. The dominant peak is at around $10^{3} \mathrm{pc} \mathrm{cm}^{-3}$.

The model that assumes a spiral host galaxy similar to the MW is modelled with two distribution functions of the position of the FRB progenitor, one is Uniform and the other follows the star density in the MW. The bulk of both of these distributions is similar to the MW. There is less $\mathrm{DM}_{\text {host }}$ around $10^{3} \mathrm{pc} \mathrm{cm}^{-3}$, since there are less LoSs that traverse big parts of the galaxy. For the Uniform distribution, a lot of progenitors are located close to the border of their host. A huge number of LoSs traverse only small parts of the galaxy. Therefore, the tail towards lower values is much more pronounced.

Xu \& Han (2015) also investigate a spiral galaxy. The maximum, $\approx 1500 \mathrm{pc} \mathrm{cm}^{-3}$, and most probable values, $\approx$ few $\mathrm{pc} \mathrm{cm}^{-3}$, are similar to our results.

The likelihood function for starburst $d w a r f$ galaxies as FRB hosts shows a flat plateau at $\mathrm{DM}_{\text {host }}=1-10^{3} \mathrm{pc} \mathrm{cm}^{-3}$ due to the assumed flat prior. In most cases, the contribution will be significantly lower than the IGM. However, there is a small probability of a few per cent that it contributes more to the $\mathrm{DM}_{\mathrm{EG}}$.

The uniform model described by Piro \& Gaensler (2018) strongly depends on the ISM density $n_{\mathrm{ISM}}$. The shape of the likelihood function is almost identical to the chosen prior distribution $\pi\left(n_{\mathrm{ISM}}\right)$. Of course, this depends on the host galaxy and we will show below the result for both host galaxy models investigated in this work.

For the case of MW-like spiral galaxies, we see in Fig. 9 that the supernova remnants can provide an observed $\mathrm{DM}_{\text {prog }}$ up to several $10^{3} \mathrm{pc} \mathrm{cm}^{-3}$, even at a distance of $z=1$, if the magnetar is located in an H II region. Only the dwarf host model has very small chance to contribute similarly high values of $\mathrm{DM}_{\text {host }}$. None of the other models is able to produce such high values of DM. This shows how likelihood functions can be used to rule out contributor models for single events and, subsequently, for whole populations.

Fig. 9 also shows that a high $\mathrm{DM}_{\text {obs }}$ does not necessarily imply a high redshift, but can also be produced in the local environment of the FRB, even if the probability is rather low, $\lesssim 1$ per cent. However, if future observations reveal a significantly higher number of large $\mathrm{DM}_{\mathrm{obs}} \gtrsim 10^{3} \mathrm{pc} \mathrm{cm}^{-3}$, this would argue in favour of a population at reasonable cosmic distance, $z \gtrsim 1$.

The wind model in Piro \& Gaensler (2018) results in a rather flat distribution of $\mathrm{DM}_{\text {prog }}$ around $\left(10^{-2}\right)-\left(10^{-1}\right) \mathrm{pc} \mathrm{cm}^{-3}$ that decreases rapidly at both ends. Adding the SNR contribution in wind $+S N R$, the plateau expands to substantially higher values of $10^{1} \mathrm{pc} \mathrm{cm}^{-3}$ and the tail includes $\mathrm{DM}_{\text {prog }} \gtrsim 10^{2} \mathrm{pc} \mathrm{cm}^{-3}$ with a probability of $\approx 0.1$ per cent. At redshift $z=1$, this is far below the IGM contribution.

\subsubsection{Rotation measure}

Fig. 10 shows the likelihood functions of RM for all models at redshift $z=1$.

The model for the MW is in agreement with the data provided by Oppermann et al. (2015). The likelihood function is of similar shape as for the IGM, about an order of magnitude above the primordial model. It stays above both models of the IGM for all redshifts probed in this work, $z \leq 6$.

The host model that resembles an MW-like spiral galaxy shows a likelihood function for $\mathrm{RM}_{\text {host }}$ that is very peaked around $10^{1}$ $10^{2} \mathrm{rad} \mathrm{m}^{-2}$ - about a magnitude above the peak of the primordial model - in case the positions of FRB progenitors scale with the star 


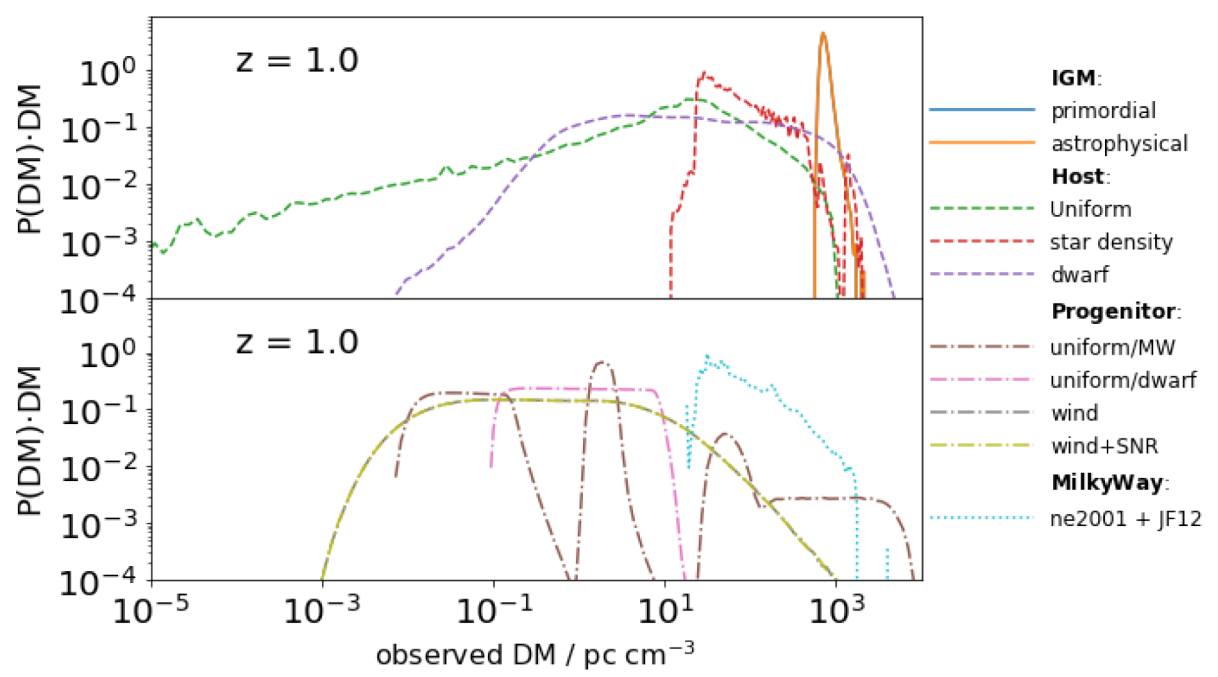

Figure 9. Likelihood functions $P(\mathrm{DM} \mid z=1)$ for all contributor models investigated in this work. The linestyle indicates the contributing region described by the model.

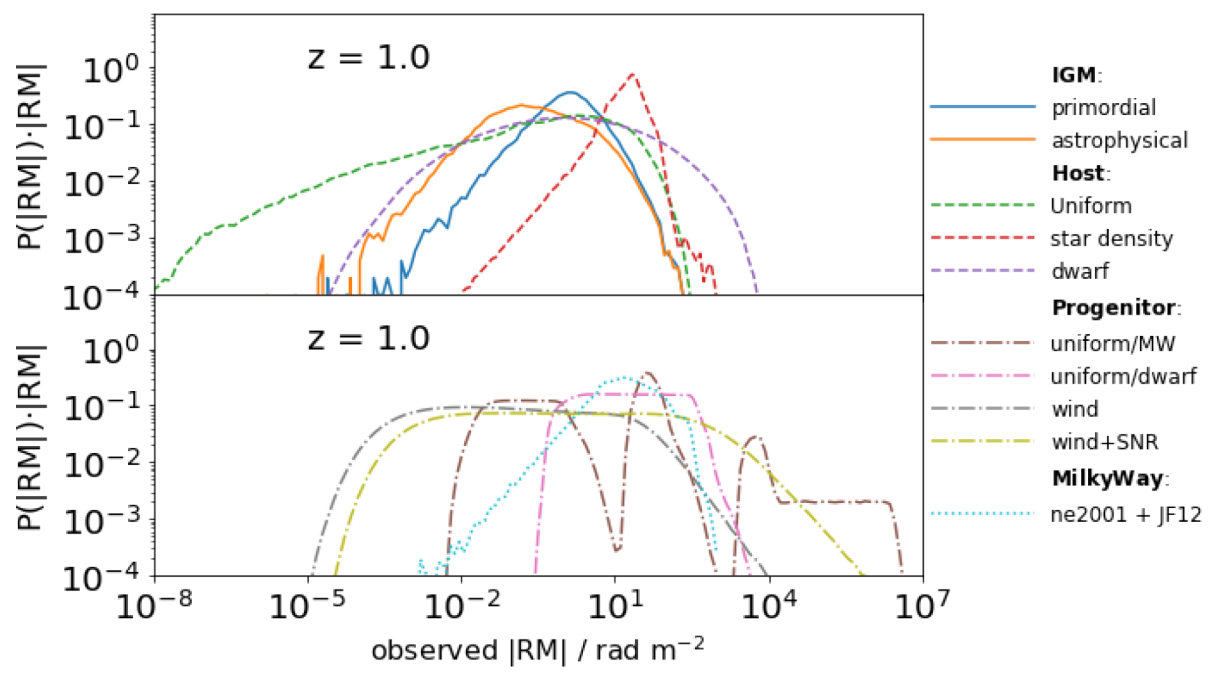

Figure 10. Likelihood functions $P(\mathrm{RM} \mid z=1)$ for all contributor models investigated in this work. The linestyle indicates the contributing region described by the model.

density. This falls off exponentially with distance from the centre of the galaxy, which hosts most candidate locations and gives the strongest contribution to $\mathrm{RM}_{\text {host }}$.

For a Uniform distribution of progenitors, there is a wide and pronounced tail towards lower values of $\mathrm{RM}_{\text {host }}$, due to the numerous short LoSs of progenitors located at the border of the galaxy. In this case, the bulk of $\mathrm{RM}_{\text {host }}$ is comparable to the IGM contribution. These models are in best agreement with the results by Basu et al. (2018), who investigated the RM contribution of a randomly orientated galaxy in the LoS of a quasar. The range up to $\lesssim$ few $100 \mathrm{rad} \mathrm{m}^{-2}$ and median $\approx 10 \mathrm{rad} \mathrm{m}^{-2}$ of their distribution is comparable to our results.

The starburst $d$ warf galaxy model assumes the distribution of progenitor positions to be concentrated close to the galactic centre. Overall, the contribution is stronger than for a Uniform distribution of sources in a spiral galaxy, since most LoSs traverse considerable portions of high-density regions in the galactic disc. Due to the small size of a dwarf galaxy, the majority of LoSs show $\mathrm{RM}_{\text {host }}$ below the most probable value found for star density distribution in spiral galaxies.

The uniform model of the local environment of neutron stars described in Piro \& Gaensler (2018) strongly depends on the local ISM density $n_{\text {ISM }}$. Hence, the shape of the likelihood function is determined by the prior distribution chosen for $n_{\text {ISM }}$ and allows us to easily associate $\mathrm{RM}_{\text {prog }}$ with the medium around the progenitor. This depends on the galaxy that hosts the FRB and we present results for both models of the host galaxy investigated in this work. In case of a spiral galaxy like the MW, we see that for magnetars located in H II regions, the contribution of the remnants of the recent supernova can reach extremely high $\mathrm{RM}_{\text {prog }}$ up to several $10^{6} \mathrm{rad} \mathrm{m}^{-2}$, exceeding RMs in that region observed with background sources by several orders of magnitude (e.g. Harvey-Smith, Madsen \& Gaensler 2011).

There is a reasonable probability of about 1 per cent to see $\mathrm{RM}_{\text {prog }}$ $\gtrsim 10^{4}-10^{5} \mathrm{rad} \mathrm{m}^{-2}$ from magnetars in these regions. This suggests that the high $\mathrm{RM}_{\mathrm{obs}}$ of FRB121102 (Michilli et al. 2018) might be the signal of an FRB located in an $\mathrm{H}$ II region. However, the bulk of 
$\mathrm{RM}_{\text {prog }}$ expected in both models is of the order of the contribution of the IGM or the MW.

If the local environment of the magnetar was instead dominated by the stellar wind of the seed star, the likelihood function of $\mathrm{RM}_{\text {prog }}$ is rather flat $\left(10^{-4}\right)-\left(10^{1}\right) \mathrm{rad} \mathrm{m}^{-2}$ with rapidly falling tails on both sides. Adding the SNR contribution in wind $+S N R$, the plateau expands to $10^{3} \mathrm{rad} \mathrm{m}^{-2}$ with a high-end tail reaching out to $10^{5} \mathrm{rad} \mathrm{m}^{-2}$. However, it barely reaches values high enough to explain the high $\mathrm{RM}_{\mathrm{obs}}$ of FRB121102. Since this model is more of an upper limit than a prediction, this scenario is highly unlikely. Therefore, the best-fitting scenario for FRB121102 from the models of this paper is a magnetar localized in an $\mathrm{H}$ II region. This is in close agreement with previous works, which concluded that FRB121102 is likely produced by a magnetar in high-density regions (Masui et al. 2015; Spitler et al. 2016; Beloborodov 2017). Note, however, that a wide range of sizes and densities of $\mathrm{H}$ II regions is excluded by constraints from DM and the absence of free-free absorption (Michilli et al. 2018).

\subsection{Dependence on redshift}

From the likelihood functions derived above, we compute the expectation value and deviation of the contributor models in order to compare their contribution at different redshifts more easily. The results are shown in Fig. 11.

The MHD simulations probed by Vazza et al. (2018) are produced in the same framework. We use similar starting parameters, adding the constrained initial conditions. The resulting LoSs are, statistically speaking, almost identical. The redshift dependence of the average $\langle\mathrm{DM}\rangle$ contribution of the IGM compares well to the results of Akahori et al. (2016). Since the other extragalactic contributions decrease with redshift, the IGM strongly dominates the total $\mathrm{DM}_{\mathrm{obs}}$ at redshifts $z \gtrsim 1$. However, there is little change in $\mathrm{DM}_{\mathrm{IGM}}$ with redshift $z>1-2$. This introduces huge uncertainties in estimating the corresponding redshift for high DMs.

At low redshifts, $z<0.1$, the IGM contribution is substantially subdominant to the contributions of the MW and the host galaxy. Hence, the $\mathrm{DM}_{\mathrm{obs}}$ can only provide an upper limit on $z$ (cf. Dolag et al. 2015; Niino 2018; Walker et al. 2018; Pol et al. 2019). The different models for progenitor environment and host galaxy do not show significant differences.

For the $\langle\mathrm{RM}\rangle$, the different models of progenitor environment and host galaxy result in rather different contributions. For example, a spiral galaxy similar to the MW on average contributes two orders of magnitude higher $\mathrm{RM}_{\text {host }}$ than a dwarf galaxy similar to IC 10 .

Regardless of the model, the contribution from the host galaxy and/or the progenitor environment dominates the $\mathrm{RM}_{\text {obs }}$ of FRBs in the local Universe $z<0.1$. The choice of models determines at which point the IGM will take over. Although the contribution of the MW is dominant at all redshifts up to $z=6$, we argue that this contribution can be removed by subtracting the MW component with sophisticated modelling of the Galaxy (Boulanger et al. 2018). At high latitudes, $\mathrm{RM}_{\mathrm{MW}} \approx 10 \mathrm{rad} \mathrm{m}^{-2}$ are still very likely. Hence, it does not suffice to restrict the sample to FRBs observed outside the Galactic plane.

The difference in average $\left\langle\mathrm{RM}_{\mathrm{IGM}}\right\rangle$ between the primordial and astrophysical models is about one order of magnitude at $z=1$. That difference increases with redshift to almost two orders of magnitude at $z=6$, where the primordial model is dominant over all other extragalactic contributions. This shows that $\mathrm{RM}_{\mathrm{EG}}$ of FRBs delivers information on and can be used to constrain the strength and origin of the IGMF. However, the minimum redshift of FRBs required to allow us to derive conclusions strongly differs for different host galaxies and progenitor environments.

\section{COMBINED RESULTS}

\subsection{Extragalactic likelihood function}

In the previous sections, we derived likelihood functions for all extragalactic contributors of $\mathrm{DM}_{\mathrm{obs}}$ and $\mathrm{RM}_{\mathrm{obs}}$ measured for FRBs. In this section, we combine these results into a likelihood function for the total extragalactic contribution. The combined likelihood function of the sum of independent variables is the convolution of their individual likelihood functions,

$P_{\mathrm{EG}}=P_{\mathrm{IGM}} * P_{\mathrm{host}} * P_{\mathrm{prog}}$.

We stress that the results presented in this section cannot yet be compared to observations directly, without assumptions on the FRB population and observational selection effects. If, for example, the number of FRBs increases with redshift, higher values of DM and $\mathrm{RM}$ are expected than for a constant number of FRBs. In the future, population assumptions and selection effects will be implemented using results of FRBPOPPY ${ }^{1}$ in order to provide detailed predictions, tailored to the individual telescope, to be compared to observations.

We compute the likelihood of the extragalactic component $\mathrm{DM}_{\mathrm{EG}}$, assuming that FRBs are produced in a wind progenitor environment hosted by a starburst $d$ warf galaxy. This set of models was chosen in order to obtain the most optimistic results on obtaining info about the IGMF. Since the density distribution is the same in the primordial and astrophysical IGM models, we only show results for the former. These are shown in Fig. 12 for FRBs at different redshifts.

As explained in the previous section, the $\mathrm{DM}_{\mathrm{EG}}$ is strongly dominated by the IGM at high redshifts $z>1$. Therefore, the combined likelihood function is almost identical to that of the IGM alone. The distribution in Fig. 12 becomes much narrower. The range reduces from over two orders of magnitude, $\sim 10^{2}-$ $10^{4} \mathrm{pc} \mathrm{cm}^{-3}$, at redshift $z=0.1$ to a range of less than factor 2 at redshift $z=6$, peaked at around $2 \times 10^{3} \mathrm{pc} \mathrm{cm}^{-3}$. The peak value is determined by the IGM and increases with redshift. The tail to high $\mathrm{RM}_{\mathrm{EG}}$, provided by strong progenitor contribution, decreases and is completely overshadowed by the IGM distribution by redshift $z \approx 1$. However, the increase of the peak value is rather slow at high redshift. This introduces a high uncertainty in determination of the exact redshift using $\mathrm{DM}_{\text {obs }}$.

The contribution of the host galaxy can cause huge values of observed $\mathrm{DM}_{\text {host }}$. which exceed the contribution of the IGM even at very high redshifts, $z \gtrsim 6$. Therefore, high DMs do not necessarily imply a high redshift of the source, but could also be produced in a nearby host galaxy. Note, though, that the likelihood of high $\mathrm{DM}_{\text {host }}$ at low $z<1$ is rather low, $\lesssim$ few per cent, as the bulk of $\mathrm{DM}_{\text {host }}$ is about an order of magnitude below results of the IGM at $z>1$. If the observed amount of FRBs with high DMs is found to be $\gtrsim 5$ per cent, this would allow us to conclude on a cosmic population $z$ $>1$.

We further compute the likelihood of the extragalactic component $\mathrm{RM}_{\mathrm{EG}}$, assuming that FRBs are produced in a wind progenitor environment hosted by a starburst dwarf galaxy. To see the difference for the scenarios of magneto-genesis of IGMFs, we compute results for

\footnotetext{
${ }^{1}$ https://github.com/davidgardenier/frbpoppy
} 

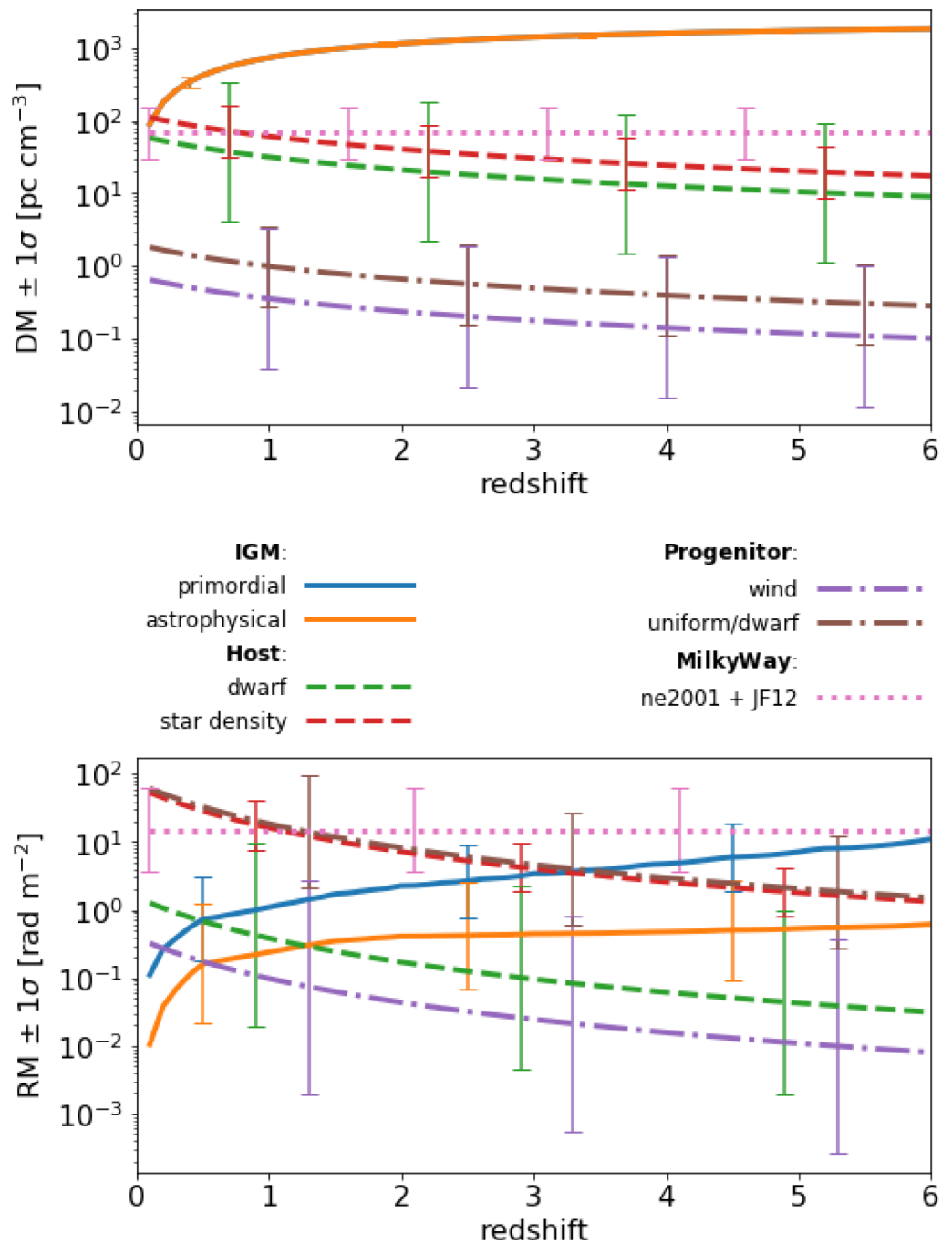

Figure 11. Redshift dependence in the distant Universe of the different average contributions $\langle\mathrm{DM}\rangle$ (top) and $\langle\mathrm{RM}\rangle$ (bottom).

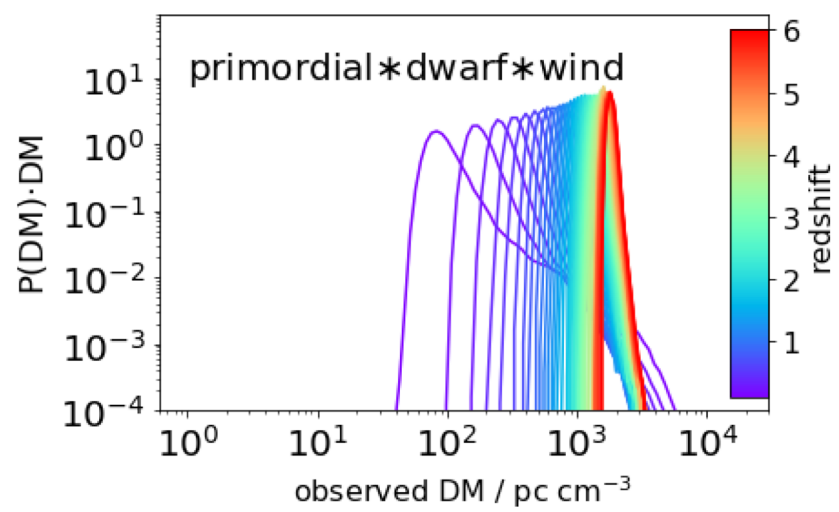

Figure 12. Combined likelihood function $P_{\mathrm{EG}}$ of all extragalactic contributors to DM, assuming that FRBs are produced at redshift $z$ in a wind progenitor environment and hosted by a starburst $d$ warf galaxy embedded in an IGMF of primordial origin. From blue to red, the graphs show results for increasing redshift in the distant Universe, $0.1 \leq z \leq 6.0$ in steps of 0.1 . both the primordial and astrophysical cases. The results for FRBs at different redshifts are shown in Figs 13 and 14.

At low redshift, the shape of $P\left(\mathrm{RM}_{\mathrm{EG}} \mid z\right)$ is determined by the host contribution. However, there is a significant difference between the two models, already at $z=0.5$, that grows with redshift, though the average of both distributions is comparable. A quantification of that difference can be found in Section 4.2.

For the primordial model, contributions from the IGM become comparable to the host contribution at $z \approx 0.5$. This allows us to lower the chance of the highest $\mathrm{RM}_{\mathrm{EG}}$ due to cancellation of $\mathrm{RM}$ from different regions, while intermediate results $\gtrsim 1 \mathrm{rad} \mathrm{m}^{-2}$ become more likely.

At higher redshift, $z \gtrsim 4$, the shape is completely determined by the IGM contribution, as it exceeds the observed contribution of the host galaxy at such high redshifts. This shows the capability of $\mathrm{RM}_{\mathrm{obs}}$ of FRBs to shed light on the origin of IGMFs.

Note that, although the values of $\mathrm{RM}_{\mathrm{IGM}}$ in the astrophysical case are equal or smaller than in the primordial case, there can be a slightly higher chance of a high $\mathrm{RM}_{\mathrm{EG}}$ in the former case. This is because RMs from different regions of the LoS, e.g. IGM and host, can cancel each other. Hence, two comparably strong contributors 


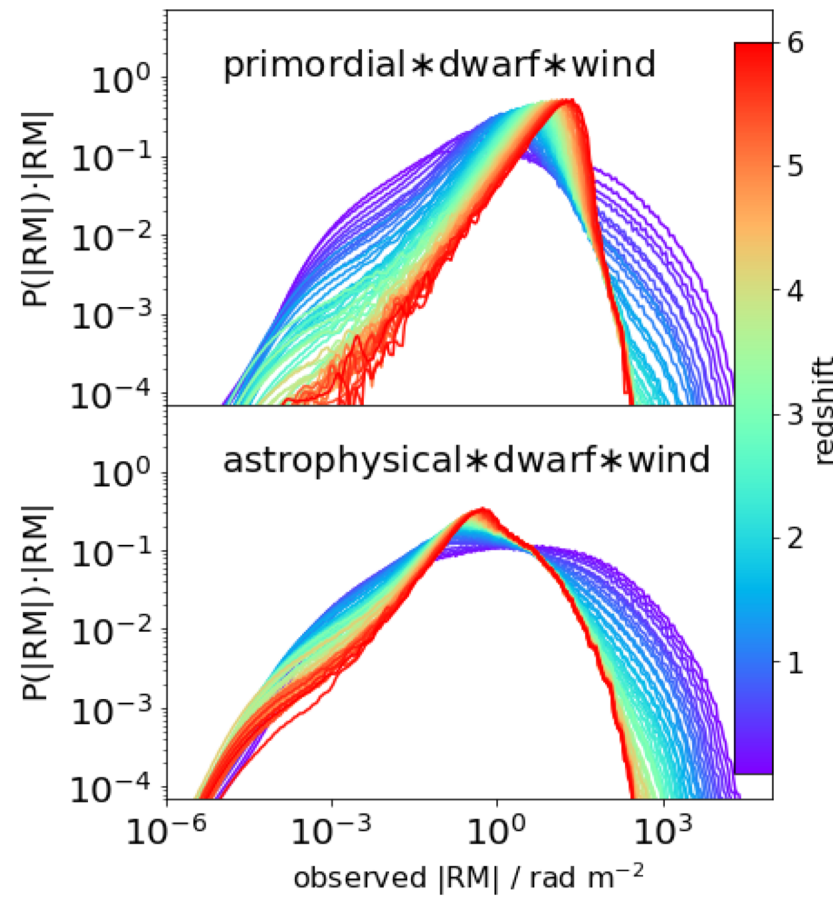

Figure 13. Combined likelihood $P_{\mathrm{EG}}$ of all extragalactic contributors to $\mathrm{RM}$, assuming that FRBs are produced at redshift $z$ in a wind progenitor environment and hosted by a starburst dwarf galaxy embedded in an intragalactic magnetic field of primordial (top) or astrophysical (bottom) origin. From blue to red, the graphs show results for increasing redshift in the distant Universe, $0.1 \leq z \leq 6.0$ in steps of 0.1 .

can weaken the likelihood for high $\mathrm{RM}_{\mathrm{obs}}$, as compared to only one dominant contributor. Use of the likelihood function can account for that, which is an advantage as compared to considering only the average value.

We stress that results in this section highly depend on the choice of contributor models. Here, we made use of those host galaxy and progenitor environment models, which showed the least contribution to $\mathrm{RM}_{\mathrm{EG}}$. We did this in order to derive the most optimistic results on obtaining info on the IGM. The results in Figs 9-11 show that the other host and progenitor models investigated here provide much higher values of RM that overshadow the IGM contribution up to redshift $z=3-4$. Ways to restrict the inference to those FRBs that fit the presented choice of models will be discussed in Section 5 and will be the subject of upcoming works.

\subsection{Application to observations}

At this point, there are few observations of FRBs with $\mathrm{RM}_{\mathrm{obs}}$. This will change soon, after new telescopes dedicated to observe FRBs, e.g. CHIME/FRB, FAST, SKA, and MeerKat, begin producing RM data (Jonas 2009; Nan et al. 2011; Keane et al. 2013; Macquart et al. 2015; The CHIME/FRB Collaboration 2018). We therefore investigate samples of random tuples of DM and RM that resemble the expected distribution at redshift $z=0.5$ shown in Figs 7 and 14. Note that contributions from the ionosphere to the RM are expected to be a few $\mathrm{rad} \mathrm{m}^{-2}$ (Weisberg et al. 2003), therefore hampers investigation of the distribution of $\mathrm{RM}_{\mathrm{obs}}<$ $1 \mathrm{rad} \mathrm{m}^{-2}$. To account for that, we only sample RM above that value.

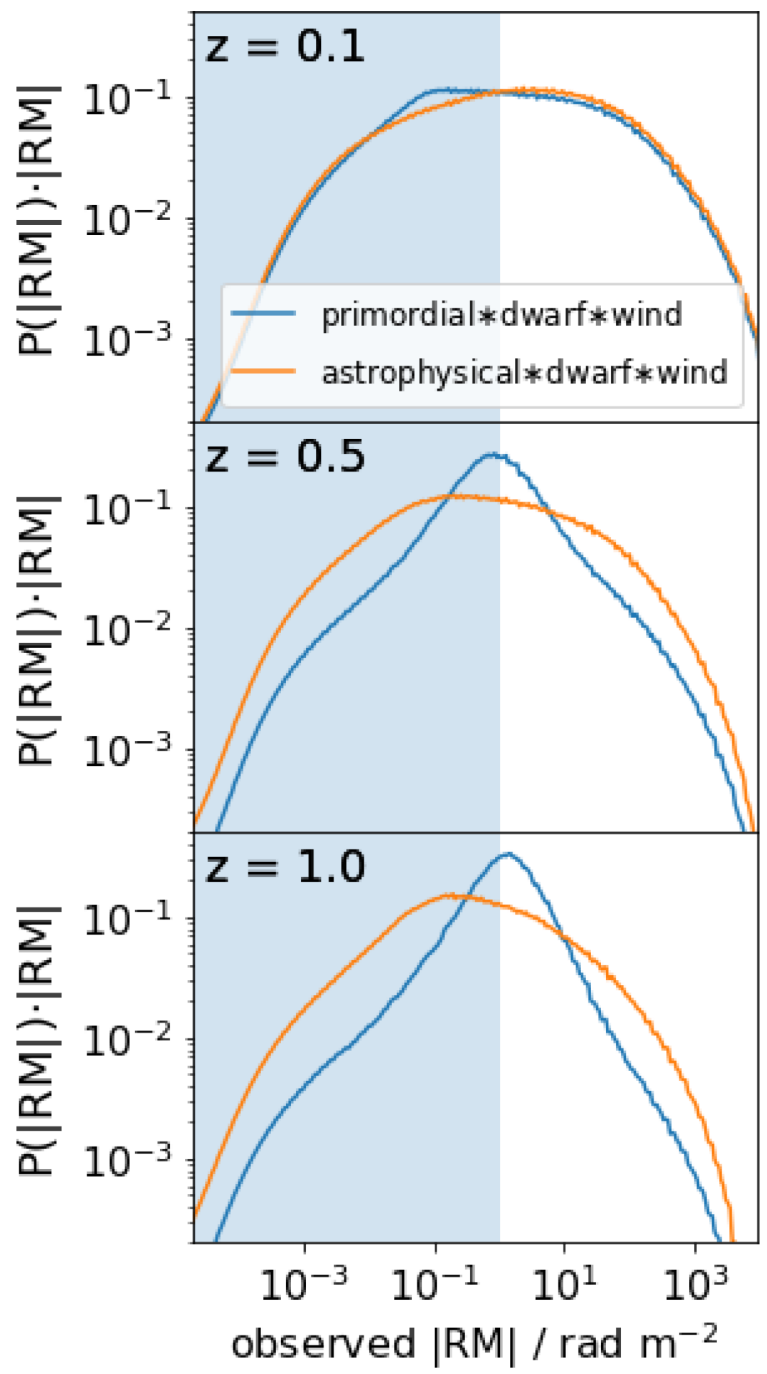

Figure 14. Same as Fig. 13 with both models in a single plot for a small set of redshifts to allow better comparison. The grey area indicates $\mathrm{RM}_{\mathrm{EG}}$ $<1 \mathrm{rad} \mathrm{m}^{-2}$ that we consider as not observable due to uncertainties in removing the foreground of the MW and ionosphere.

For each of the (DM, RM) tuples, we compute the Bayes factor

$b(\mathrm{DM}, \mathrm{RM} \mid$ primordial, astrophysical)

$$
=\frac{P(\mathrm{DM}, \mathrm{RM} \mid \text { primordial })}{P(\mathrm{DM}, \mathrm{RM} \mid \text { astrophysical })},
$$

which quantifies how much more likely it is that the given tuple of $\mathrm{DM}_{\mathrm{EG}}$ and $\mathrm{RM}_{\mathrm{EG}}$ is produced in the primordial rather than in the astrophysical case. A Bayes factor $b\left(O, M_{1}, M_{2}\right)>10$ shows that observation $O$ is more than 10 times more likely in model $M_{1}$ than in model $M_{2}$. This signals strong evidence in favour of $M_{1}$ as compared to $M_{2}$. For values $b>100$, the evidence is considered to be decisive (Jeffreys \& Jeffreys 1961).

The likelihood of two events is the product of their individual likelihoods. The same holds for the Bayes factor, which applies a number to our corroboration towards one model over the other.

In particular, we use the $\mathrm{DM}_{\mathrm{EG}}$ to derive a likelihood function $P\left(z \mid \mathrm{DM}_{\mathrm{EG}}\right)$ for the redshift of the FRB. This is then used as a prior 


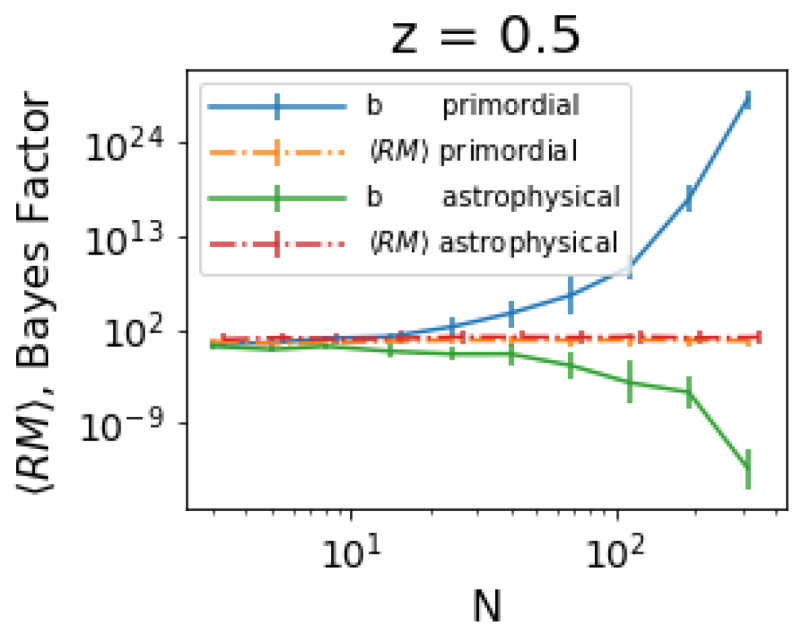

Figure 15. Bayes factor $b$ and average $\left\langle\mathrm{RM}_{\mathrm{EG}}\right\rangle$ for two fake populations at the indicated redshift that resemble the primordial and astrophysical cases. The errorbars of $\left\langle\mathrm{RM}_{\mathrm{EG}}\right\rangle$ show the $1 \sigma$ standard deviation of $\mathrm{RM}$ in the population. For $b$, they show the standard deviation of six random samples of the population.

to derive the likelihood of the $\mathrm{RM}_{\mathrm{EG}}$

$P\left(\mathrm{DM}_{\mathrm{EG}}, \mathrm{RM}_{\mathrm{EG}} \mid B O\right) \propto \int P\left(\mathrm{RM}_{\mathrm{EG}} \mid B O, z\right) P\left(z \mid \mathrm{DM}_{\mathrm{EG}}\right) \mathrm{d} z$.

Note that this inference does not require knowledge on the redshift of the $\mathrm{FRB}$, but only uses the $\mathrm{DM}_{\mathrm{EG}}$ and $\mathrm{RM}_{\mathrm{EG}}$. If the redshift is known, $P\left(z \mid \mathrm{DM}_{\mathrm{EG}}\right)$ can be replaced by a narrower function in order to decrease the range of possible $\mathrm{RM}_{\mathrm{EG}}$ in the different models and allow for more precise results.

Note that RMs from AGNs are much easier to be associated with a redshift. Hence, including AGNs in our analysis in future work will significantly improve the results of this section, despite the missing DM.

We compute the Bayes factor for different sizes of the sample to see how many FRBs are required at a given redshift in order to allow conclusions on the IGM. We also compute the average $\left\langle\mathrm{RM}_{\mathrm{EG}}\right\rangle$ of these samples to compare the efficiency of the Bayesian inference to the frequentists' approach. The results are shown in Fig. 15.

The $\left\langle\mathrm{RM}_{\mathrm{EG}}\right\rangle$ agree within $1 \sigma$ standard deviation for both populations, whereas the Bayes factor shows a difference $\gtrsim 10$ orders of magnitude for a number of 100 FRBs at redshift $z=0.5$, in case they are produced by magnetars in wind environments hosted by dwarf galaxies. This huge difference clearly sets apart the scenarios for the generation of the IGMF.

We stress that this result is largely dependent on the assumed model for the host galaxy and progenitor environment since Fig. 11 shows that other choices lead to very different results. This can shift the required redshift of FRBs, e.g. hosted by spiral galaxies, to much higher redshifts, $z \gtrsim 3$ or even above. Hence, an identification of the host galaxy as well as the local environment of the progenitor is crucial for their use to probe IGMFs. This can be a difficult task, especially for the case of dwarf galaxies (e.g. Eftekhari \& Berger 2017).

We compare several possible combinations of models to investigate the redshift of FRB sources required to obtain information on the IGMF in those scenarios. We sample $\mathrm{DM}_{\mathrm{EG}}$ and $\mathrm{RM}_{\mathrm{EG}}$ of 100 FRBs, all at the same redshift and compute the corresponding Bayes factor $b(\mathrm{DM}, \mathrm{RM} \mid$ primordial, astrophysical) (equation 8). This procedure is then repeated with increasing redshift. We compute six random samples at each of these redshifts and plot the average and standard deviation of the Bayes factor. The results are shown in Fig. 16.

For three of the seven combinations of models, at redshifts $z \gtrsim$ 0.5 , the resulting Bayes factor is $b \gg 10^{2}$ and hence clearly speaks for a primordial origin of IGMFs (in case of a primordial fake population. The same holds for the astrophysical scenario.). These are the combinations that assume the wind or wind $+S N R$ model for the progenitor together with a dwarf host galaxy. The wind model delivers a distribution of $\mathrm{RM}_{\text {prog }}$ that is more concentrated on lower values as compared to the uniform model. Though the former delivers much higher values of $\mathrm{RM}_{\text {prog }}$, this is mostly for times $t \lesssim 25 \mathrm{yr}$, below which the radio bursts are weakened by the supernova ejecta (Margalit et al. 2018). At later times, the predicted $\mathrm{RM}_{\text {prog }}$ decreases much faster in the wind than in the uniform case, accounting for the higher amount of low $\mathrm{RM}_{\text {prog }}$ in the former case.

The third combination considers both galaxy models, the $d w a r f$ as well as the MW-like spiral galaxy, star density, as equally likely hosts. This is done by using the renormalized sum of both likelihood functions, shown in Figs 9 and 10. The resulting distribution is much less peaked than the star density case and tends to lower values, therefore enabling those FRBs to deliver information on the IGM. This means that, even if not all of the FRBs taken into account are hosted by $d$ warf galaxies, their overall statistics at redshift $z \gtrsim 0.5$ may still allow us to draw conclusions on the magneto-genesis of IGMFs.

We note that the equal weighting of the two host models is an arbitrary choice, not based on any realistic population synthesis of galaxies. In reality, the weighting for different types of galaxies changes with redshift, as does the galactic stellar mass function (e.g. Lilly et al. 2009) as well as the major star population and their age in different types of galaxies (e.g. Hopkins 2004). An increase of the weight of spiral galaxies, star density, would increase probability of strong host contributions and hence push the redshift required to probe the IGM to higher values. Future work should account for that by assuming several possible populations of FRBs, their possible host galaxies and redshift distribution.

Four of the seven combinations result in indecisive Bayes factors, $b \lesssim 10^{2}$, at redshifts below $z \lesssim 3$. These are scenarios that assume either an MW-like galaxy, star density, as the host of FRBs with any model for the progenitor investigated in this work, or a magnetar embedded in a uniform environment hosted by a dwarf galaxy. In these cases, the local contribution is too strong to allow us to infer information on the IGM. For the spiral galaxy model, the distribution of $\mathrm{RM}_{\text {host }}$ peaks at about $\gtrsim 10^{1} \mathrm{rad} \mathrm{m}^{-2}$ in the host rest frame. This strong contribution can overshadow the contribution from the IGM at high redshift. However, even for these unfavourable models, beyond redshifts of $z \gtrsim 3.5-4$, the contribution of the IGM becomes strong enough to allow to distinguish between different scenarios of magneto-genesis of IGMFs. FRBs at such high redshift probably will not even require us to select a special subset of the population, e.g. hosted by dwarf galaxies, in order to obtain reasonable results. However, using only FRBs beyond redshift $z \sim$ 4 might be even harder to accomplish, as it is a tough task, in case they exist, to find the exact redshift of FRBs at this distance. On the one hand, the DM can only be used to derive an upper limit on $z$, as we show in Section 4. On the other hand, identification of the a dwarf host galaxy becomes increasingly harder with growing redshift (e.g. Eftekhari \& Berger 2017).

Note that the $d$ warf*uniform case shows vastly lower values of the Bayes factor at redshifts $z>4$ than all of the star density combinations. The latter are dominated by the host contribution 


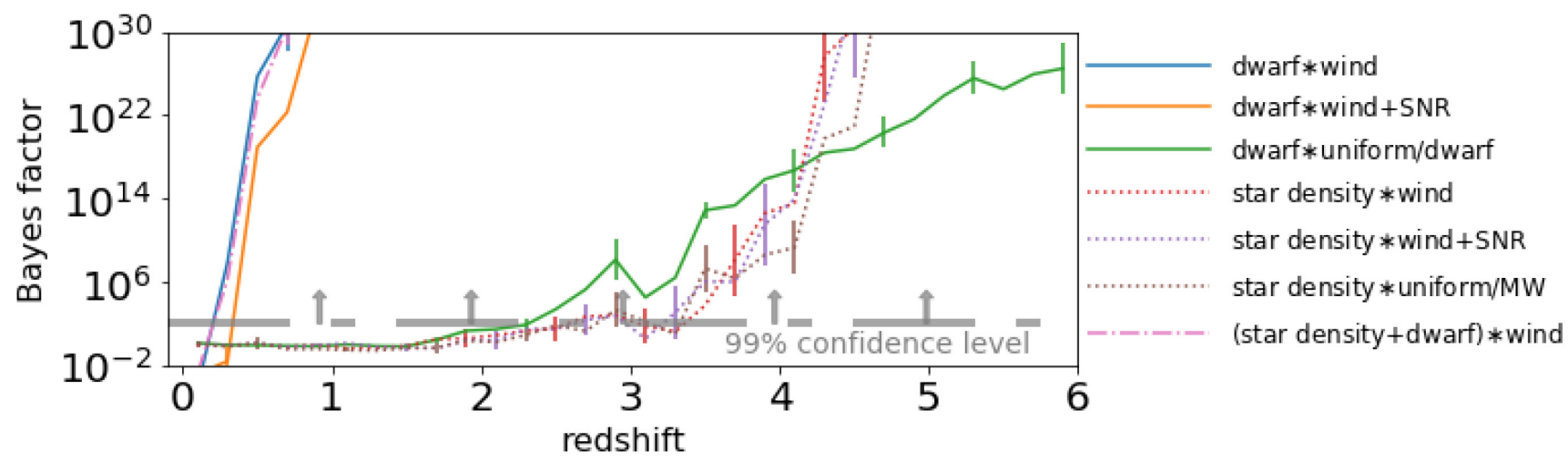

Figure 16. Bayes factor for fake samples of $100 \mathrm{FRBs}$ at different redshifts, resembling the population in the primordial magneto-genesis scenario, combined with several sets of models indicated by colours and linestyles. The solid lines consider a $d w a r f$ galaxy as the host of FRBs, the dotted lines assume the MW-like spiral galaxy, star density, while the dash-dotted line allows for both of these galaxy types to host similar numbers of FRBs. The grey line marks a Bayes factor of $10^{2}$, which indicates a 100 times higher chance for the fake population to be produced in the primordial rather than in the astrophysical scenario, assuming the same models for the other contributors. This indicates the 99 per cent confidence level to rule out the latter scenario in favour of the former.

for all models of the progenitor environment. The very narrow distribution $P\left(\mathrm{RM}_{\text {host }} \mid\right.$ star density $)$ peaks between the primordial and astrophysical $P\left(\mathrm{RM}_{\mathrm{IGM}}\right)$. Their convolution, $P_{\mathrm{Host}} * P_{\mathrm{IGM}}$, is rather different for the two cases.

In contrast, the dwarf*uniform case is dominated by the local environment of the progenitor, which shows a very flat distribution, $P\left(\mathrm{RM}_{\text {prog }}\right)$, due to the assumed flat prior. The primordial $P\left(\mathrm{RM}_{\mathrm{IGM}}\right)$ peaks within the range of $P\left(\mathrm{RM}_{\text {prog }}\right)$; their convolution, $P\left(\mathrm{RM}_{\mathrm{EG}}\right)$, has similar shape to $P\left(\mathrm{RM}_{\text {prog }}\right)$, altered only by a subtle peak at high values. The primarily low contributions of the astrophysical $\mathrm{RM}_{\mathrm{IGM}}$ do not alter the shape of $P\left(\mathrm{RM}_{\text {prog }}\right)$ significantly. Hence, the full likelihood functions $P\left(\mathrm{RM}_{\mathrm{EG}}\right)$ have similar shape for both models of the IGM. Single events have less weight as evidence because the Bayes factor is generally closer to unity. In mathematical terms, the integral over the absolute difference of the likelihood functions of the two cases is higher for the star density galaxy combinations than for the dwarf*uniform case, which is hence less informative. Note that this is another measure that might be used to infer the likelihood of different combinations of models.

Note further that for all four of these models, the Bayes factor $b$ drops significantly at around $z \approx 3$. This is because the shapes of $P\left(\mathrm{RM}_{\text {host }} \mid\right.$ star density $)$ and $P\left(\mathrm{RM}_{\mathrm{IGM}} \mid\right.$ primordial $)$ are almost identical, as their peaks move through the same value at this redshift. This causes the two contributions to greatly match each other, resulting in an identical shape of the full $P\left(\mathrm{RM}_{\mathrm{EG}}\right)$. In contrast to that, $\mathrm{RM}_{\mathrm{IGM}}$ values in the astrophysical scenario are too weak to significantly alter the shape of $P\left(\mathrm{RM}_{\text {host }}\right)$. Hence, the two IGM scenarios appear very similar at that redshift. This cosmic conspiracy might be used to infer the strength of the primordial magnetic field $B$, as the position of the dip highly depends on $B$. However, it also strongly depends on the shape of the contribution of the host galaxy and might not be visible for other sets of models.

We have shown that there likely exists at least a subset of FRBs - produced by magnetars in wind environments hosted by starburst dwarf galaxies - that carries information on the IGMF. However, other host galaxies and progenitor classes completely overshadow that signal of the IGM. This shows how important it is to carefully consider the numerous possible models for regions along the LoS and to identify the host galaxies and source objects in order to measure the IGMF using FRBs.

According to the Bayes' theorem, in order to arrive at the ratio of posterior likelihoods $L$ for the different models, the Bayes factor $B$ has to be multiplied by the prior corroboration $\pi(M)$ towards a model $M$, based on information other than the investigated observation $O$ (e.g. Boulanger et al. 2018; Fraix-Burnet et al. 2018):

$$
\frac{L\left(M_{1} \mid O\right)}{L\left(M_{2} \mid P\right)}=b\left(O \mid M_{1}, M_{2}\right) \times \frac{\pi\left(M_{1}\right)}{\pi\left(M_{2}\right)} .
$$

Note that we assume the two IGM models to be equally likely, i.e. $\pi=$ const. It therefore suffices to investigate the Bayes factor $b$ to infer the posterior likelihood of different models.

\section{DISCUSSION}

We investigate whether observations of FRBs can deliver information about the IGMF and its origins. To this end, we consider two extreme scenarios of magneto-genesis: a scenario where the IGMF is seeded at very high redshift (termed primordial scenario) and a second scenario where the magnetic field is mainly supplied by galactic outflows and other astrophysical processes (termed astrophysical scenario).

The initial magnetic field is very different in the two scenarios. Hence, the two scenarios account for a strong difference in the strength of magnetic fields far outside the overdense regions in the Universe. This implies significantly different results for the RM and makes these two suitable models to investigate the potential of FRBs to probe the IGMF. We compute likelihood functions of these measures that allow a comparison of the assumed models to observational data.

To account for the contribution towards the rotation and dispersion measures of the host, we investigated two models for the host galaxy, i.e. an MW-like spiral galaxy and a starburst dwarf galaxy similar to IC 10 or the host of FRB121102. This only serves as an illustration of our framework to compare theory and observations and this framework can easily be expanded to include a large variety of models for the host galaxy. Results agree with previous works (Xu \& Han 2015; Basu et al. 2018).

Likewise, we model in Section 2.3 the contribution of the local environment of the progenitor with Monte Carlo simulations using the results of Piro \& Gaensler (2018). The source of FRBs is assumed to be a magnetar embedded either in a uniform ISM or an environment disturbed by stellar winds of the seed star. For the uniform case, the distribution of possible ISM number densities $n_{\text {ISM }}$ is determined by the host galaxy. 
In accordance with previous work (Dolag et al. 2015; Niino 2018; Walker et al. 2018; Pol et al. 2019), we find that the DM is an imprecise measure of the source redshift and only delivers reasonable upper limits. Only few of the investigated models had low probability to supply DM in excess of $\mathrm{DM}_{\mathrm{IGM}}$. Hence, a significant fraction $\gtrsim 5$ per cent of high $\mathrm{DM}_{\mathrm{obs}} \gtrsim 10^{3} \mathrm{pc} \mathrm{cm}^{-3}$ would point to a population of FRBs at cosmic distance, $z \gtrsim 1$. However, this requires a more detailed investigation that takes into account the uncertain evolution of the number of FRB sources with redshift, as well as the selection effects of the telescopes. We note that this is the scope of FRBPOPPY!, ${ }^{2}$ the results of which will be implemented in this framework in the future.

Bhandari et al. (2017) report three FRBs with very high $\mathrm{DM}_{\text {obs }}>$ $1500 \mathrm{pc} \mathrm{cm}^{-3}$ detected by the Parkes telescope. Although the DMs are highly debated to be produced locally, they raise hope that there is indeed an FRB population at large distance that will be detected in the years to come. For example, the MeerKat and Parkes telescopes can detect FRBs out to redshift $z \approx 4$ (Keane 2018). ARECIBO may detect bursts at $z \approx 5$ (Lorimer 2018), while FAST will be able to detect FRBs even out to $z \approx 15$ (Zhang 2018), with $\mathrm{DM}_{\mathrm{obs}}$ exceeding $10^{4} \mathrm{pc} \mathrm{cm}^{-3}$. Our study shows that these FRBs are an interesting source of information on the IGMF and its origins.

For the limited set of models investigated in this paper, only a few progenitor models are capable to produce the high RM observed for FRB121102 (Michilli et al. 2018). For other FRBs with less extreme RMs, conclusions on their source are less obvious and require careful investigation of the convolved likelihood functions of the different contributors. The time evolution of repeating FRBs can be used to put much better constraints on the source model. This is, however, beyond the scope of this study and will be considered in upcoming work.

The models applied for the host galaxy use analytic functions and do not account for local overdensities, which can add significantly to $\mathrm{RM}_{\text {host }}$. Also, our models of the host do not yet account for cosmic evolution of the galaxy. Results of Pillepich et al. (2019) suggest that low-mass star-forming galaxies do not change their size significantly out to redshift $z=4-5$. Hence, the values of $\mathrm{DM}_{\text {host }}$ are not expected to change much for the dwarf-type of galaxies considered here. They further find that massive galaxies similar to the MW tend to be smaller at higher redshift. The density can be higher by a factor of few tens, while the path-length is reduced by a factor of a few, accounting for a DM higher by about one order of magnitude than predicted in our model, still mostly below the contribution of the IGM.

For magnetic fields in galaxies, the amplification time is of the order of $10^{7}-10^{8} \mathrm{yr}$ (e.g. Schober, Schleicher \& Klessen 2013). Observations and simulations of galaxies at high redshift suggest magnetic fields of similar strength as in present-day galaxies (Bernet et al. 2008; Kronberg et al. 2008; Pakmor, Marinacci \& Springel 2014; Mao et al. 2017). Hence, the expected change to the $\mathrm{RM}_{\text {host }}$ is of the same order as for the $\mathrm{DM}_{\text {host }}$, insignificant for dwarf-type and about one order of magnitude higher for galaxies similar to the MW. This implies that the latter type of host dominates the extragalactic contribution and does not allow for conclusions on the IGMF, even out to redshift $z=6$. However, Rodrigues et al. (2018) conclude that a significant fraction of massive spiral galaxies contain negligible large-scale magnetic fields at redshifts $z>3$, suggesting a significantly weaker host contribution. A more physical modelling of the host galaxies will be the subject of future work.

${ }^{2}$ https://github.com/davidgardenier/frbpoppy
We account for effects from the possible progenitor positions by testing different distributions within the host galaxy. We find that assuming a uniform distribution in the host disc affects the distribution of expected $\mathrm{DM}_{\text {host }}$ only at values $\lesssim$ few $\mathrm{pc} \mathrm{cm}^{-3}$, as compared to a distribution that concentrates on the centre of the galaxy.

The distribution of expected $\mathrm{RM}_{\text {host }}$ is very different, even $\gtrsim 10^{1} \mathrm{rad} \mathrm{m}^{-2}$, close to the maximum possible value, with a much higher probability in the centred case because the highest $\mathrm{RM}_{\text {host }}$ come from the centre of the galaxy. We note, however, that our model does not include the high $\mathrm{RM} \approx-5.6 \times 10^{5} \mathrm{rad} \mathrm{m}^{-2}$ found for Sagittarius $A^{\star}$ (Marrone et al. 2006). Such contributions from a LoS through the galactic centre of the host galaxy might explain the high RM observed for FRB121102 (Michilli et al. 2018). We argue that such LoSs are very unlikely for progenitors that are not themselves located in the centres of their host galaxy.

By assuming only magnetars as progenitors, we restrict the parameter space for equations in Piro \& Gaensler (2018), as compared to neutron stars with weaker magnetic fields, e.g. pulsars. By that, we mostly exclude lower values of $R M_{\text {prog }}$, hence arrive at rather optimistic predictions for the contribution from the local environment of the progenitor. Further, their model assumes that shocks in supernova remnants produce coherent magnetic fields, while it most likely has a random topology. Hence, the results for the uniform and the wind $+S N R$ model should be considered as upper limits. In case the real contribution of RM from such magnetars is significantly lower, these sources might also deliver information on the IGMF.

We do not account for the contributions of the MW halo. For the DM, they are comparable to the contribution from the Galactic disc, $\approx 30-80 \mathrm{pc} \mathrm{cm}^{-3}$ (Dolag et al. 2015; Prochaska \& Zheng 2019). The $\mathrm{RM}$ from the halo is probably lower than from the disc, due to the weaker magnetic fields. However, the likelihood function of the two models for the IGM show reasonable difference at redshift $z=0.5$ even for $\mathrm{RM}_{\mathrm{EG}}>1 \mathrm{rad} \mathrm{m}^{-2}$. We only used $\mathrm{RM}_{\mathrm{EG}}$ above this value in estimates of the model likelihood in Section 4.2.

We do not account for the distribution of galaxies that host FRBs. By applying a constant weight to each LoS, we implicitly assume a uniform distribution of host galaxies. Reducing the weight of LoS through low-density regions mostly reduces the likelihood of low values of RM that cannot be probed by telescopes. Estimating the effect on likelihood of $\mathrm{RM} \gtrsim 1 \mathrm{rad} \mathrm{m}^{-2}$ is not trivial and will be studied in upcoming works. However, for FRBs beyond redshift $z$ $\gtrsim 0.1$, the overall statistics are not expected to change, since the Universe is homogeneous on large scales.

We do not account for the contribution of intervening galaxies (e.g. Basu et al. 2018). If the intervening galaxy is of the same type as the host, the contribution is comparable to the host contribution at the redshift of intersection and therefore hampers the investigation of the IGM component (Zheng et al. 2014). If the pulse broadening of FRB radiation is found to be dominated by scattering in intervening galaxies, this can help to exclude LoS with a significant contribution of intervening galaxies (Lorimer et al. 2013; Spitler et al. 2014). This will be a subject of future studies.

Our results are provided in the form of likelihood functions for the different contributions to RM and DM. We show how these likelihood functions can be used for parameter inference. This framework can help to infer the origin of FRBs as well as the origin of extragalactic magnetic fields (Caleb et al. 2018; Katz 2018; Palaniswamy et al. 2018). 


\section{CONCLUSIONS}

In this paper, we have studied the different contributions to the DMs and RMs along the lines of sight of FRBs. We have built a Bayesian framework to interpret observable information of FRBs. We show how this can be used to constrain the amplitude of magnetic fields in the IGM along the line of sight. Our key findings are as follows:

(i) The strengths of the different contributions to the observed $\mathrm{RM}_{\mathrm{obs}}$ highly depend on the assumed model for FRBs and their host galaxies. Magnetars embedded in wind environments hosted by starburst dwarf galaxies provide the lowest average local contribution to RM of the investigated models. For this generous set of models, $\mathrm{RM}_{\mathrm{EG}}$ from redshifts $z \gtrsim 0.5$ can potentially provide information on the magnetic field in the IGM and its origin. At this redshift, the contribution of the IGM is still subdominant to that of the host. Still, there is a significant change in the distribution of extragalactic $\mathrm{RM}_{\mathrm{EG}}$. This allows one to draw conclusions on the magnetic field using Bayesian inference.

Conversely, for other models of the host galaxy and progenitor, the expected local contribution can be significantly stronger. These models require FRBs beyond $z \gtrsim 3$ in order to probe IGMFs. We conclude that there are good reasons to believe that at least a subset of FRBs observed with RMs delivers information on the IGMF and its origin. How to identify this subset will be subject of future studies.

(ii) The MW provides the dominant contribution of RM, even for FRBs out to redshift $z \gtrsim 6$. A prerequisite for the above result is the removal of the contribution of the MW to a precision of $\sim 1 \mathrm{rad} \mathrm{m}^{-2}$. This is non-trivial, as argued by Han (2017), who suggested that up to $\sim 10^{4}-10^{5}$ values of RM may be necessary to tell apart Galactic from extragalactic contributions. However, the fast growing level of complexity in modelling magnetic fields in the MW is expected to improve at the same pace as RM statistics, making the removal of the MW foreground more viable (for a recent review, see e.g. Boulanger et al. 2018).

(iii) Using likelihood functions allows one to infer information on the host galaxy and progenitor. They allow us to systematically rule out models for a single FRB or groups of those.

(iv) From the present set of models, only some progenitor models are capable of producing the very high $\mathrm{RM}_{\mathrm{obs}}$ of FRB121102. Our results suggest that, if the progenitor is a magnetar, then it is most likely located in a dense environment, such as an $\mathrm{H}$ II region, which we found to be capable of producing RMs that exceed those of FRB121102 by two orders of magnitude. Note, however, that the strong magnetic fields generated by shocks in the supernova remnants are likely random. This can result in much lower RM than predicted by the model of Piro \& Gaensler (2018), who assumed a coherent magnetic field.

We find a much smaller chance that stellar winds of the seed star in other environments can account for the high $\mathrm{RM}_{\mathrm{obs}}$ as well. The shape of magnetic fields induced by stellar winds is very coherent and can account for very high values of RM. However, the expected $\mathrm{RM}_{\text {prog }}$ falls rapidly with age of the magnetar. This implies a much lower chance to observe high $\mathrm{RM}_{\text {prog }}$ from such a source.

We provide a framework for the comparison between observations and theories of FRBs. So far, we consider a very limited set of models in order to present our framework. Still, we could show the likely existence of a subset of FRBs that delivers information on the IGMF and its origins. Future work will include more models, such as elliptical or disc host galaxies, and take into account their evolution with redshift. We will vary the strength of a primordial magnetic field in realistic combination with astrophysical processes. This will allow us to precisely probe the average strength of IGMFs today as well as the strength of the primordial seed field, thus allow us to constrain processes of magneto-genesis with FRBs.

At this point, we only consider the DM and RM. In future work, more observables will be considered, such as temporal scattering, flux density, and fluence. Further contributing regions will be considered, such as intervening galaxies and the halo of the MW. Combining this with knowledge on the selection effect of telescopes and assumptions on the underlying population of FRBs, we can produce individual predictions for the distribution of observables as measured at different telescopes. ${ }^{3}$

\section{ACKNOWLEDGEMENTS}

We thank Tony Piro for his help in understanding and reproducing results from his paper. We also thank Ryan Mckinven for interesting comments. Further, we thank the anonymous referee for a detailed review and constructive comments. SH would like to thank all participants of 'A Bayesian View on the Galactic Magnetic Field' as well as the Lorentz Center for an inspiring meeting. SH would also like to thank the organizers, speakers, and participants of the 'Stat4Astro School of Statistics for Astrophysics 2017: Bayesian Methodology' and the 'FRB2019' conference in Amsterdam for interesting discussions.

Our cosmological simulations were performed with the ENZO code (http://enzo-project.org), under project $\mathrm{HHH} 38$ and $\mathrm{HHH} 42$ at the Jülich Supercomputing Centre (P.I. F. Vazza). FV acknowledges financial support from the ERC Starting Grant 'MAGCOW', no. 714196. We thank Jenny Source and Stefan Gottlöber for providing us with the CLUES initial conditions and for their help in implementation.

The Dunlap Institute is funded through an endowment established by the David Dunlap family and the University of Toronto. BMG acknowledges the support of the Natural Sciences and Engineering Research Council of Canada (NSERC) through grant RGPIN-201505948, and of the Canada Research Chairs programme.

\section{REFERENCES}

Ade P. et al., 2016, A\&A, 594, A19

Akahori T., Ryu D., Gaensler B. M., 2016, ApJ, 824, 105

Baldry I. et al., 2012, MNRAS, 421, 621

Basu A., Mao S. A., Fletcher A., Kanekar N., Shukurov A., Schnitzeler D., Vacca V., Junklewitz H., 2018, MNRAS, 477, 2528

Beloborodov A. M., 2017, ApJ, 843, L26

Beloborodov A. M., Li X., 2016, ApJ, 833, 261

Beniamini P., Hotokezaka K., van der Horst A., Kouveliotou C., 2019, MNRAS, 487, 1426

Bernet M. L., Miniati F., Lilly S. J., Kronberg P. P., Dessauges-Zavadsky M., 2008, Nature, 454, 302

Bhandari S. et al., 2017, MNRAS, 475, 1427

Boulanger F. et al., 2018, J. Cosmol. Astropart. Phys., 2018, 049

Bryan G. L. et al., 2014, ApJS, 211, 19

Caleb M., Spitler L. G., Stappers B. W., 2018, Nat. Astron., 2, 839

Caleb M., Stappers B. W., Rajwade K., Flynn C., 2019, MNRAS, 484, 5500

Campanelli L., 2009, Int. J. Mod. Phys. D, 18, 1395

Chabrier G., 2003, PASP, 115, 763

${ }^{3}$ This is the aim of the PREFRBLE ('Predictions of FRB models and their Likelihood Estimates') python package that comes with the presented results for a fast application to observational data. This package is currently under construction and will be publicly available soon. 
Chabrier G., 2005, in Corbelli E., Palle F., eds, The Initial Mass Function 50 Years Later. Springer, Dordrecht, p. 41

Chatterjee S. et al., 2017, Nature, 541, 58

CHIME/FRB Collaboration, 2019, Nature, 566, 235

Chyży K. T., Drzazga R. T., Beck R., Urbanik M., Heesen V., Bomans D. J., 2016, ApJ, 819, 39

Cordes J. M., Lazio T. J. W., 2002, astro-ph/0207156

da Silva A. C., Barbosa D., Liddle A. R., Thomas P. A., 2000, MNRAS, 317,37

de Avillez M. A., Breitschwerdt D., 2005, A\&A, 436, 585

Deng W., Zhang B., 2014, ApJ, 783, L35

Derman E., Demircan O., Kahraman G., 1990, A\&AS, 86, 421

Dolag K., Gaensler B. M., Beck A. M., Beck M. C., 2015, MNRAS, 451, 4277

Donnert J., Dolag K., Lesch H., Müller E., 2009, MNRAS, 392, 1008

Dubois Y., Teyssier R., 2010, A\&A, 523, A72

Dzhatdoev T., Khalikov E., Kircheva A., Podlesnyi E., Telegina A., 2018, in Volkova V.E., Zhezher Y. V., Levkov D. G., Rubakov V. A., Matveev V. A., eds, EPJ Web Conf., Vol. 191, Intergalactic Electromagnetic Cascades in the Magnetized Universe as a Tool of Astroparticle Physics. EDP Sci. - Web Conf., Les Ulis Cedex A, France, p. 08009

Eftekhari T., Berger E., 2017, ApJ, 849, 162

Feretti L., Giovannini G., Govoni F., Murgia M., 2012, A\&AR, 20, 54

Ferrière K. M., 2001, Rev. Mod. Phys., 73, 1031

Fraix-Burnet D., Girard S., Arbel J., Marquette J.-B., 2018, Statistics for Astrophysics: Bayesian Methodology. EDP Sci., Les Ulis Cedex A, France, https://hal.archives-ouvertes.fr/hal-01952759

Fruchter A. et al., 2006, Nature, 441, 463

Górski K. M., Hivon E., Banday A. J., Wandelt B. D., Hansen F. K., Reinecke M., Bartelmann M., 2005, ApJ, 622, 759

Hackstein S., Vazza F., Brüggen M., Sorce J. G., Gottlöber S., 2018, MNRAS, 475, 2519

Han J. L., 2017, ARA\&A, 55, 111

Harvey-Smith L., Madsen G. J., Gaensler B. M., 2011, ApJ, 736, 83

Heesen V., Rau U., Rupen M., Brinks E., Hunter D. A., 2011, ApJ, 739, L23

Hessels J. et al., 2019, ApJ, 876, L23

Hopkins A. M., 2004, ApJ, 615, 209

Houben L. J. M., Spitler L. G., ter Veen S., Rachen J. P., Falcke H., Kramer M., 2019, A\&A, 623, A42

Hummels C. B., Smith B. D., Silvia D. W., 2017, ApJ, 847, 59

Inoue S., 2004, MNRAS, 348, 999

Ioka K., 2003, ApJ, 598, L79

James C. W., 2019, MNRAS, 486, 5934

Jansson R., Farrar G. R., 2012, ApJ, 757, 14

Jeffreys H., Jeffreys H., 1961, Theory of Probability, 3rd edn. Clarendon, OUP Oxford, 1998

Jonas J. L., 2009, Proc. IEEE, 97, 1522

Jurić M. et al., 2008, ApJ, 673, 864

Kahniashvili T., Tevzadze A. G., Sethi S. K., Pandey K., Ratra B., 2010, Phys. Rev. D, 82, 083005

Katz J. I., 2016a, Mod. Phys. Lett. A, 31, 1630013

Katz J. I., 2016b, ApJ, 826, 226

Katz J. I., 2018, Prog. Part. Nucl. Phys., 103, 1

Keane E. et al., 2016, Nature, 530, 453

Keane E., 2018, Nat. Astron., 2, 1811

Keane E. F., Fender R. P., Hassall T. E., 2013, MNRAS, 436, 371

Kronberg P., Bernet M., Miniati F., Lilly S., Short M., Higdon D., 2008 , ApJ, 676, 70

Kronberg P. P., Lesch H., Hopp U., 1999, ApJ, 511, 56

Kumar P., Linder E. V., 2019, preprint (arXiv:1903.08175)

Leroy A., Bolatto A., Walter F., Blitz L., 2006, ApJ, 643, 825

Li B., Li L.-B., Zhang Z.-B., Geng J.-J., Song L.-M., Huang Y.-F., Yang Y.-P., 2019, preprint (arXiv:1901.03484)

Lilly S. J. et al., 2009, ApJS, 184, 218

Locatelli N., Ronchi M., Ghirlanda G., Ghisellini G., 2019, A\&A, 625, A109

Lorimer D., Bailes M., McLaughlin M., Narkevic D., Crawford F., 2007, Science, 318, 777
Lorimer D. R., 2018, Nat. Astron., 2, 860

Lorimer D. R., McLaughlin M. A., Karastergiou A., Johnston S., 2013, MNRAS, 436, L5

Lu W., Kumar P., Narayan R., 2018, MNRAS, 483, 359

Luo R., Lee K., Lorimer D. R., Zhang B., 2018, MNRAS, 481, 2320

Lyutikov M., 2019, preprint (arXiv:1901.03260)

Macquart J. P. et al., 2015, Proc. Sci., Fast Transients at Cosmological Distances with the SKA. SISSA, Trieste, PoS\#55

Magrini L., Gonçalves D. R., 2009, MNRAS, 398, 280

Mao S. et al., 2017, Nat. Astron., 1, 621

Marcote B., Paragi Z., 2019, preprint (arXiv:1901.08541)

Margalit B., Metzger B. D., Berger E., Nicholl M., Eftekhari T., Margutti R., 2018, MNRAS, 481, 2407

Marrone D. P., Moran J. M., Zhao J.-H., Rao R., 2006, ApJ, 654, L57

Masui K. et al., 2015, Nature, 528, 523

McQuinn M., 2013, ApJ, 780, L33

Metzger B. D., Berger E., Margalit B., 2017, ApJ, 841, 14

Metzger B. D., Margalit B., Sironi L., 2019, MNRAS, 485, 4091

Michilli D. et al., 2018, Nature, 553, 182

Nan R. et al., 2011, Int. J. Mod. Phys. D, 20, 989

Neronov A., Vovk I., 2010, Science, 328, 73

Niino Y., 2018, ApJ, 858, 4

Oppermann N. et al., 2015, A\&A, 575, A118

Pakmor R., Marinacci F., Springel V., 2014, ApJ, 783, L20

Palaniswamy D., Li Y., Zhang B., 2018, ApJ, 854, L12

Pen U.-L., Connor L., 2015, ApJ, 807, 179

Perley D. A. et al., 2016, ApJ, 830, 13

Petroff E., Hessels J. W. T., Lorimer D. R., 2019, A\&AR, 27, 4

Pillepich A. et al., 2019, preprint (arXiv:1902.05553)

Piro A. L., Gaensler B. M., 2018, ApJ, 861, 150

Planck Collaboration I, 2014, A\&A, 571, A1

Platts E., Weltman A., Walters A., Tendulkar S., Gordin J., Kandhai S., Physics Reports (2019)

Pol N., Lam M. T., McLaughlin M. A., Lazio T. J. W., Cordes J. M., 2019, preprint (arXiv:1903.07630)

Popov S. B., Postnov K. A., 2010, Evolution of Cosmic Objects through their Physical Activity. Proceedings of the Conference dedicated to Viktor Ambartsumian's 100th Anniversary ed HA Harutyunian, AM Mickaelian, Y. Terzian (Yerevan: NAS RA). Vol. 129. 2010. p. 129

Prochaska J. X., Zheng Y., 2019, MNRAS, 485, 648

Ravi V. et al., 2016, Science, 354, 1249

Ravi V. et al., 2019, Bull. Am. Astron. Soc., 51, 420

Ravi V., Loeb A., 2018, ApJ, 874, 72

Richer M. et al., 2001, A\&A, 370, 34

Rodrigues L. F. S., Chamandy L., Shukurov A., Baugh C. M., Taylor A. R., 2018, MNRAS, 483, 2424

Salpeter E. E., 1955, ApJ, 121, 161

Schober J., Schleicher D. R., Klessen R. S., 2013, A\&A, 560, A87

Scholz P. et al., 2016, ApJ, 833, 177

Siegel M., Majewski S., Reid I., Thompson I., 2002, ApJ, 578, 151

Sorce J. G. et al., 2016, MNRAS, 455, 2078

Spitler L. et al., 2014, ApJ, 790, 101

Spitler L. et al., 2016, Nature, 531, 202

Stasyszyn F., Nuza S. E., Dolag K., Beck R., Donnert J., 2010, MNRAS, 408, 684

Subramanian K., 2016, Rep. Prog. Phys., 79, 076901

Taylor A., Vovk I., Neronov A., 2011, A\&A, 529, A144

Tendulkar S. P. et al., 2017, ApJ, 834, L7

The CHIME/FRB Collaboration, 2018, ApJ, 863, 48

Trivedi P., Subramanian K., Seshadri T. R., 2014, Phys. Rev. D, 89, 043523

Tully R. B. et al., 2013, AJ, 146, 86

Vallée J., 1990, AJ, 99, 459

Vazza F., Brüggen M., Gheller C., Hackstein S., Wittor D., Hinz P. M., 2017, Class. Quantum Gravity, 34, 234001

Vazza F., Brüggen M., Hinz P. M., Wittor D., Locatelli N., Gheller C., 2018, MNRAS, 480, 3907

Vergani S. et al., 2015, A\&A, 581, A102 
Waelkens A., Jaffe T., Reinecke M., Kitaura F., Enßlin T., 2009, A\&A, 495, 697

Walker C. R. H., Ma Y.-Z., Breton R. P., 2018, preprint (arXiv:1804.01548)

Weisberg J. M., Cordes J. M., Kuan B., Devine K. E., Green J. T., Backer D. C., 2004, ApJS, 150(1), 317

Wickramasinghe D., Ferrario L., 2008, MNRAS, 389, L66

Widrow L. M., 2002, Rev. Mod. Phys., 74, 775

Wit W. d., Testi L., Palla F., Zinnecker H., 2005, Astron. Astrophys.-Les Ulis, 437, 247

Woosley S., Weaver T., 1995, No. UCRL-ID-122106, Lawrence Livermore National Lab., CA (United States)

Xu J., Han J., 2014, MNRAS, 442, 3329

Xu J., Han J. L., 2015, Res. Astron. Astrophys., 15, 1629

Yang Y.-H., Zhang B.-B., Zhang B., 2019, ApJ, 875, L19

Yao J., Manchester R., Wang N., 2017, ApJ, 835, 29

Zhang B., 2014, ApJ, 780, L21

Zhang B., 2018, ApJ, 867, L21

Zheng Z., Ofek E., Kulkarni S., Neill J., Juric M., 2014, ApJ, 797, 71

\section{APPENDIX A: UNIFORM PRIMORDIAL MAGNETIC FIELD}

In this section, we briefly investigate the effects of choosing a uniform primordial magnetic field on the resulting RM likelihood functions. To this end, we compare the six models presented by Hackstein et al. (2018). In this paper, we used their primordial model, which starts with a uniform magnetic field. The other primordial models start from a purely turbulent field using different power-law indices, while the astrophysical models use a very faint seed field and instead allow for magnetic feedback from an AGN.

Using the uniform resolution grid at $z=0$, we calculate RM for LoS parallel to two axes, positive and negative directions, to obtain both signs for RM values. From that, we obtain likelihood function of RM for the different configuration of initial magnetic field.

The result in Fig. A1 shows that for the primordial model, which starts from a completely uniform magnetic field, there is a pronounced peak at around $10^{-3} \mathrm{rad} \mathrm{m}^{-2}$. The other primordial $2 R$ and primordial $3 R$ models start from a stochastic field, so contributions along the LoS can cancel out each other, and the likelihood reduces at the peak value and increases at lower values. Higher values, contributed by denser structures, are not affected by the shape of the primordial field. However, this feature is visible in the IGM component at order $\mathrm{RM}_{\mathrm{IGM}} \approx 10^{-4} \mathrm{rad} \mathrm{m}^{-2}$, and hence not accessible. This implies that RMs of FRBs do not carry information on the coherence length of primordial fields.

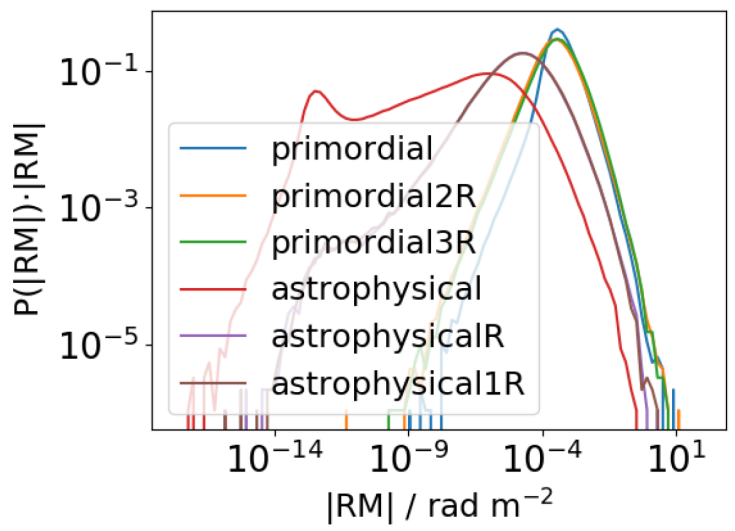

Figure A1. Likelihood function $P(\mathrm{RM})$ for the different models of the local Universe from Hackstein et al. (2018).
The astrophysical models show similar highest values of $\mathrm{RM}_{\mathrm{IGM}}$ to the primordial ones. The bulk of values is a few orders of magnitude below the primordial peak and the low tail reaches to substantially smaller values. The shape at low values is rather different from the results in Fig. 8 at redshift $z=1$, as it reaches to smaller values and peaks again at around $10^{-13} \mathrm{rad} \mathrm{m}^{-2}$. The difference is because predictions in this work have been reconstructed from the primordial model. However, since the difference in results is for values of RM that are far too low to be measurable, we consider the data sufficient for the argument of this work.

\section{APPENDIX B: PRIORS}

We perform Monte Carlo simulations in order to obtain likelihood functions for the contribution of the progenitor and the host galaxy. This requires a choice of reasonable prior probability distribution of the parameters that enter the equations. All parameters and their priors are summarized in Table B1.

We assume the source of FRBs to be magnetars, which stem from B- and O-type stars with masses of $m=20-45 \mathrm{M}_{\odot}$ and $B_{\star}=$ $800-1500 \mathrm{G}$ dipole magnetic fields. This assumption is reasonable, according to the results of Wickramasinghe \& Ferrario (2008). The $\mathrm{DM}$ and RM contribution for this case of the local environment is given by Piro \& Gaensler (2018).

Woosley \& Weaver (1995) showed that such supernovae explode with a typical energy of $E=1.2 \times 10^{51} \mathrm{erg}$, which we adopt as a constant value.

The mass of neutron stars is about $M_{\mathrm{NS}} \approx 1.5 \mathrm{M}_{\odot}$, regardless of the progenitor stars mass. Hence, the mass of supernova ejecta is the mass of the progenitor star $m$ minus the mass of the neutron star. The prior of the mass of the progenitor star is given by the initial mass function, well approximated by the Salpeter function $\pi(M) \propto M^{-2.35}$ (Salpeter 1955; Chabrier 2003, 2005), and has a support in the mass range stated above, reduced by the mass of the neutron star.

We obtain the stellar radius from the radius-mass relation of heavy stars given in Derman, Demircan \& Kahraman (1990).

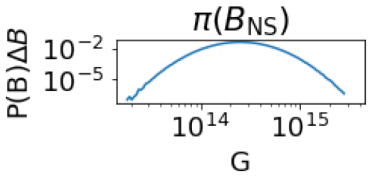

$[\mathrm{t}]$
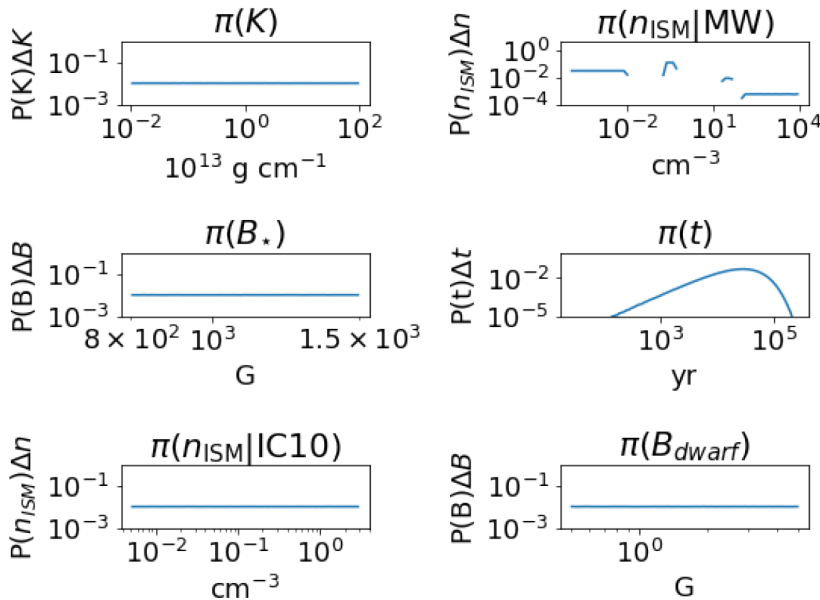

Figure B1. Graphical depiction of the priors in the Table B1. 
Table B1. Parameters for Monte Carlo simulations, and their prior distributions together with a reference.

\section{Priors:}

\section{Host galaxy}

Position of progenitor pos

Magnetic field of host $B_{\text {host }}$

\section{Progenitor}

Magnetic field of magnetar $B_{\mathrm{NS}}$

Mass of SN ejecta $M$

ISM number density $n_{\text {ISM }}$

Time since $\mathrm{SN} t$

Wind mass-loading parameter $K$

Magnetic field of seed star $B_{\star}$

\author{
MW: $\prod_{i \in[\text { thin,thick }]} \mathrm{e}^{-\frac{z}{Z_{i}}} \mathrm{e}^{-\frac{r}{R_{i}}}$ \\ IC10: $\mathrm{e}^{-\frac{z}{Z}} \mathrm{e}^{-\frac{r}{R}}$ \\ IC10: log-flat, $B_{\text {host }} \in[0.5,5] \mu \mathrm{G}$
}

$\operatorname{LogNorm}\left(\log \left(2.5 \times 10^{14} \mathrm{G}\right), 0.5\right)$

$M=m^{-2.35}-M_{\mathrm{NS}}, m \in\{20,45\} \mathrm{M}_{\odot}$

$M W: \sum \frac{p_{i}}{\Delta n}\left(\Theta\left(n-n_{i}\right)-\Theta\left(n_{i+1}-n\right)\right)$

IC10: $\log$-flat, $n_{\text {ISM }} \in\left[5 \times 10^{-3}, 3\right]$

Flat, $t \in\left\{25 \mathrm{yr}, t_{\mathrm{diss}}\right\}$

Log-flat, $K \in\left\{10^{11}, 10^{15}\right\} \mathrm{g} \mathrm{cm}^{-1}$

Log-flat, $B_{\star} \in\{800,1500\} \mathrm{G}$
Siegel et al. (2002); Jurić et al. (2008)

Leroy et al. (2006)

Chyży et al. (2016)

Wickramasinghe \& Ferrario (2008)

Chabrier (2003)

Ferrière (2001)

de Avillez \& Breitschwerdt (2005)

Margalit et al. (2018); Beloborodov \& Li (2016)

Wickramasinghe \& Ferrario (2008)
The number density of the ISM, $n_{\mathrm{ISM}}$, in an MW-like galaxy is highly varied across the different media found throughout the galaxy. We use the ranges of $n_{\text {ISM }}$ given by Ferrière (2001) together with the well-known volume filling factors of the different media. Within each of these ranges, we choose a log-flat distribution, renormalized, such that the integral over the range gives the volume filling factor of the corresponding medium.

For IC10, we assume a constant $n_{\mathrm{ISM}}$ throughout the disc of the dwarf galaxy that falls exponentially with scale height. Since FRBs are mostly located in the disc, we use identical priors for the progenitor and the host galaxy.

In general, the production of FRBs is not related to the supernova that gives birth to the magnetar. Hence, no age of the magnetar is preferred over another, which is reflected by a flat prior. Freefree absorption by supernova ejecta can weaken FRB radiation. This implies a lower limit of $t \gtrsim 25 \mathrm{yr}$ on the age of magnetars to emit visible FRBs (Margalit et al. 2018). We adopt this value as a strict lower limit. The activity period of magnetars is limited by the dissipation of their strong magnetic field, $10^{14}-10^{16} \mathrm{G}$. The dissipation time-scale was derived by Beloborodov \& Li (2016):

$t_{\text {diss }}=600\left(\frac{L}{1 \mathrm{~km}}\right)^{1.6}\left(\frac{\delta B_{\mathrm{NS}}}{10^{16} \mathrm{G}}\right)^{0.4}\left(\frac{B_{\mathrm{NS}}}{10^{16} \mathrm{G}}\right)^{-1.6}\left(\frac{\rho}{\rho_{\text {nuc }}}\right)^{1.2} \mathrm{yr}$,

where $L$ is the typical scale of variation, $\delta B_{\mathrm{NS}}$, of the magnetar's magnetic field strength, $B_{\mathrm{NS}} . \rho$ is the density of the magnetar and $\rho_{\text {nuc }}=2.8 \times 10^{14} \mathrm{~g} \mathrm{~cm}^{-3}$ is the nuclear saturation density. While the magnetic field dissipates, FRBs become less likely. We account for that by sampling possible values of $t_{\text {diss }}$ and use the shape above the maximum of the resulting probability density function.

The dissipation time, $t_{\text {diss }}$, depends on parameters that are independent of all other parameters of interest. To sample $t_{\text {diss }}$, we assume typical values of $L=10^{5} \mathrm{~cm}$ and $\rho=10^{14} \mathrm{~g} \mathrm{~cm}^{-3}$ (Beloborodov \& Li 2016). For the magnetic field of the magnetar, $B_{\mathrm{NS}}$, we roughly fit the results of Wickramasinghe \& Ferrario (2008) with a $\operatorname{LogNorm}$ function centred at $2.5 \times 10^{14} \mathrm{G}$.

The wind mass-loading parameter, $K$ (Piro \& Gaensler 2018), is not well constrained so far. Hence, we choose a log-flat prior in the expected range $K=10^{11}-10^{15} \mathrm{~g} \mathrm{~cm}^{-1}$.

The distribution of strong magnetic fields in B- and O-type stars, $B_{\star}$, is rather uncertain, as is the relation between the magnetic field of the progenitor star and that of the magnetar. This is because the strong field of the magnetar could stem from either a fossil field or a shear-driven dynamo. As a conservative choice, we use a log-flat prior for $B_{\star}$ and consider $B_{\star}$ and the magnetic field of the magnetar, $B_{\mathrm{NS}}$, as independent.

By definition, $B_{\mathrm{NS}}=10^{14}-10^{16} \mathrm{G}$. Wickramasinghe \& Ferrario (2008) give the distribution of $B_{\mathrm{NS}}$ for observed magnetars and their best-fitting model. In order to minimize selection effects from observations, we adopt their best-fitting model as a LogNorm with a mean $\mu=2.5 \times 10^{14} \mathrm{G}$ and a logarithmic deviation $\sigma=0.5$.

For simplicity, we assume that $\delta B_{\mathrm{NS}} \sim B_{\mathrm{NS}}$, which is generally the case for magnetic fields of such strength (see e.g. Beloborodov \& Li 2016).

For the host galaxy, we investigate two different models: an MWlike spiral galaxy and a dwarf galaxy similar to IC10.

We assume that the probability for the position of an FRB scales with the number density of stars. In the MW-like galaxy, the bestfitting model is the combination of two discs, thin and thick, with exponential decay from centre towards the borders. We use the bestfitting parameters for the MW, given in Jurić et al. (2008), $Z_{\text {thin }}=$ $0.3 \mathrm{kpc}, R_{\text {thin }}=2.6 \mathrm{kpc}, Z_{\text {thick }}=0.9 \mathrm{kpc}$, and $R_{\text {thick }}=3.6 \mathrm{kpc}$.

The distribution of stars in dwarf galaxies like IC10 is irregular. We hence use a simple disc model with a scale height $Z=300 \mathrm{kpc}$ (Leroy et al. 2006) and a radius $R=900 \mathrm{kpc}$.

de Avillez \& Breitschwerdt (2005) provide a distribution of ISM density, $n_{\mathrm{ISM}}$, in star-forming galaxies that is well described by a log-flat distribution and can be used as prior distribution of $n_{\text {ISM }}$ in dwarf galaxies like IC10.

Chyży et al. (2016) give a range of possible strengths for the ordered magnetic field of the dwarf galaxy IC10. We do not assume a particular shape, as the number of values is too low to derive a reasonable distribution. Hence, we use a log-flat distribution that covers the range of these values.

At this point, we take all our models as candidates with equal prior likelihood. The ratio of their inferred posteriors is hence equal to the ratio of their measure likelihoods, i.e. the Bayes factor.

This paper has been typeset from a $\mathrm{T}_{\mathrm{E}} \mathrm{X} / \mathrm{LT} \mathrm{E} \mathrm{X}$ file prepared by the author. 